\title{
Madelung representation of damped parametric quantum oscillator and exactly solvable Schrödinger-Burgers equations
}

\author{
Şirin A. Büyükaşık ${ }^{a)}$ and Oktay K. Pashaev ${ }^{\text {b) }}$ \\ Department of Mathematics, Izmir Institute of Technology, 35430 Urla, Izmir, Turkey
}

(Received 25 May 2010; accepted 11 November 2010; published online 30 December 2010)

\begin{abstract}
We construct a Madelung fluid model with time variable parameters as a dissipative quantum fluid and linearize it in terms of Schrödinger equation with time-dependent parameters. It allows us to find exact solutions of the nonlinear Madelung system in terms of solutions of the Schrödinger equation and the corresponding classical linear ordinary differential equation with variable frequency and damping. For the complex velocity field, the Madelung system takes the form of a nonlinear complex Schrödinger-Burgers equation, for which we obtain exact solutions using complex Cole-Hopf transformation. In particular, we give exact results for nonlinear Madelung systems related with Caldirola-Kanai-type dissipative harmonic oscillator. Collapse of the wave function in dissipative models and possible implications for the quantum cosmology are discussed. (c) 2010 American Institute of Physics. [doi:10.1063/1.3524505]
\end{abstract}

\section{INTRODUCTION}

In the recent years, the Madelung fluid description of quantum mechanics has been applied to some fields where the quantum formalism is a useful tool for describing the evolution of classical (quantumlike) systems and studying the dispersionless or semiclassical limit of nonlinear partial differential equations of Schrödinger type, Ref. 1. The Madelung fluid representation, proposed first by Madelung, Ref. 2, being a complex quantity, represents a solution of the Schrödinger equation, in terms of modulus and phase. Substituted to the Schrödinger equation, it allows to obtain a pair of nonlinear hydrodynamic-type equations. Then the principle of probability conservation in quantum mechanics becomes related with a hydrodynamic interpretation of continuity equation for probability flow. This hydrodynamic formulation offers an opportunity to connect directly the motion of quantum particles with the motion of a fluid and gives a different way to visualize the quantum mechanical evolution, Ref. 3. Moreover, the Madelung fluid representation becomes fundamental in superconductivity theory, Ref. 4, and description of quantum fluids like superfluid He, Ref. 5. In such models, Madelung hydrodynamic variables have direct physical meaning, as $\rho=|\Psi|^{2}$ plays the role of the superfluid density, while $v=\nabla(\arg \Psi)$ becomes the superfluid velocity.

Here, we would like to emphasize a remarkable property of the Madelung transformation. The Madelung fluid equations are nonlinear system of partial differential equations (PDEs), while the Schrödinger equation is the linear one. Then, the Madelung transform is a complex linearization transform, similar to the Cole-Hopf transformation, Refs. 6 and 7, linearizing the nonlinear Burgers equation in terms of the linear heat equation. Nonlinear models admitting such type of direct linearization are called by Calogero as $C$-integrable models, Ref. 8. From this point of view, the Madelung nonlinear fluid equations are $C$-integrable.

It is an interesting problem to describe dissipation in quantum fluids and the Madelung representation could be very useful in this study. Recently, the quantum mechanical description of

\footnotetext{
a) Author to whom correspondence should be addressed. Electronic mail: sirinatilgan @iyte.edu.tr.

b) Electronic mail: oktaypashaev@iyte.edu.tr.
} 
dissipative systems has attracted a lot of attention. These systems can be implemented as a particular realization of the time-dependent Schrödinger equation with time variable parameters. In the case of parametric harmonic oscillator with time-dependent frequency and mass, the exact solution of the Schrödinger equation can be given in terms of solution of the corresponding classical linear ordinary differential equation, Refs. 9-12. In the present paper, we construct the Madelung fluid model with time variable parameters as a dissipative quantum fluid and linearize it in terms of the time variable Schrödinger equation. It allows us to find exact solution of the nonlinear Madelung system in terms of solutions of the Schrödinger equation and the corresponding classical damped parametric oscillator. The Madelung system written for the complex velocity field takes the form of a nonlinear Burgers-type equation, which we call the complex Schrödinger-Burgers equation. Then, we use complex Cole-Hopf transformation to obtain exact solutions of nonlinear dissipative complex Schrödinger-Burgers models. As known, in the usual Cole-Hopf transformation zeros of the linear heat equation lead to poles in the corresponding Burgers equation. Similarly, in our case, by the complex Cole-Hopf transformation the zeros of the Schrödinger equation transform to pole singularities in the complex Schrödinger-Burgers equation. Thus, using the exact solutions of the linear problem, we describe also the dynamics of the poles in the corresponding nonlinear problem.

We would like to remark here that in the study of the Schrödinger equation, it is common to look for solutions in a Hilbert space and corresponding physical interpretation of the quantum mechanics. However, considering the linear Schrödinger equation as linearization of the nonlinear Madelungtype PDE, solutions of the Schrödinger equation which are not square integrable, not physically meaningful from the point of view of quantum mechanics, can also lead to an important class of solutions to the related nonlinear problems. As we mentioned above, in this case the hydrodynamic system has meaning with direct physical interpretation.

The paper is organized as follows. In Sec. II, first we give exact solutions of the linear Schrödinger equation for harmonic oscillator with time-dependent parameters in terms of solution of the corresponding classical parametric oscillator, see (Ref. 9). Then, we find the Madelung representation of the Schrödinger equation as a pair of time variable quantum Hamilton-Jacobi and continuity equation. The hydrodynamic form of the equations with variable coefficients are found as well. For each nonlinear system, we construct an exact formal solution with general initial conditions and explicit solution for some specific initial conditions. In Sec. III, we formulate the Madelung system as the complexified quantum Hamilton-Jacobi equation in the form of a potential Schrodinger-Burgers equation and using the results in Sec. II, we find exact solutions of the corresponding initial value problem (IVP). In Sec. IV, using the complex Cole-Hopf transformation, we obtain exact solutions of the complex Schrödinger-Burgers equation with time variable parameters in terms of solutions to the corresponding linear Schrödinger equation. This leads also to exact solutions for the system of nonlinear PDE's for the classical velocity and quantum velocity. In Sec. V, we describe in details a dissipative Madelung fluid and the nonlinear complex Schrödinger-Burgers model related with Caldirola-Kanai oscillator, when mass of the system is exponentially growing with time, Refs. 13 and 14. Exact solutions of the nonlinear models are found for the critical, underdamped and the overdamped cases. Moreover, the trajectories describing the motion of zeros and poles are derived and given explicitly. For each case, some illustrative plots are constructed. Section VI includes some remarks on the probability density functions of the Caldirola-Kanai oscillator, which behave like delta-convergent sequences at time infinity and show some unusual properties at the support of test functions. Section VII is the conclusion part.

\section{SCHRÖDINGER EQUATION FOR HARMONIC OSCILLATOR WITH TIME-DEPENDENT PARAMETERS AND ITS MADELUNG REPRESENTATION}

Consider the one-dimensional Schrödinger equation with time-dependent parameters

$$
i \hbar \frac{\partial \Psi}{\partial t}=-\frac{\hbar^{2}}{2 \mu(t)} \frac{\partial^{2} \Psi}{\partial q^{2}}+\frac{\mu(t) \omega^{2}(t)}{2} q^{2} \Psi
$$


and initial condition

$$
\Psi\left(q, t_{0}\right)=\psi(q), \quad-\infty<q<\infty .
$$

Using the evolution operator method it was proved that, see (Ref. 9), if $x(t)$ is the solution of the classical equation of motion

$$
\ddot{x}+\frac{\dot{\mu}(t)}{\mu(t)} \dot{x}+\omega^{2}(t) x=0, x\left(t_{0}\right)=x_{0} \neq 0, \dot{x}\left(t_{0}\right)=0,
$$

with $\mu(t)>0, \mu(t) \in C^{\prime}(I), \omega^{2}(t)>0, \omega(t) \in C(I), t_{0} \in I \subset R$, then for all $t \in I$ such that $x(t) \neq 0$, the solution of the quantum evolution problem (1)-(2) is given by $\Psi(q, t)=\widehat{\mathcal{U}}\left(t, t_{0}\right) \psi(q)$, where the evolution operator is

$$
\widehat{\mathcal{U}}\left(t, t_{0}\right)=\exp \left(\frac{i}{2} f(t) q^{2}\right) \exp \left(h(t)\left(q \frac{\partial}{\partial q}+\frac{1}{2}\right)\right) \exp \left(-\frac{i}{2} g(t) \frac{\partial^{2}}{\partial q^{2}}\right),
$$

and the auxiliary functions are

$$
f(t)=\frac{\mu(t)}{\hbar} \frac{\dot{x}(t)}{x(t)} ; \quad g(t)=-\hbar x^{2}\left(t_{0}\right) \int^{t} \frac{d \xi}{\mu(\xi) x^{2}(\xi)}, \quad g\left(t_{0}\right)=0 ; \quad h(t)=\ln \frac{\left|x\left(t_{0}\right)\right|}{|x(t)|} .
$$

If the initial function $\psi(q)$ belongs to the Hilbert space $L_{2}(R)$, then it can be written in the form $\psi(q)=\sum_{k=0}^{\infty}\left\langle\psi, \varphi_{k}\right\rangle \varphi_{k}(q)$, where

$$
\varphi_{k}(q)=N_{k} e^{-\frac{\Omega_{0}}{2} q^{2}} H_{k}\left(\sqrt{\Omega_{0}} q\right), \quad k=0,1,2, \ldots,
$$

are the normalized eigenstates corresponding to eignenvalues $E_{k}=\hbar^{2} \Omega_{0}(k+1 / 2)$ of the Hamiltonian for the standard harmonic oscillator:

$$
\widehat{H}_{0}=-\frac{\hbar^{2}}{2} \frac{\partial^{2}}{\partial q^{2}}+\frac{\omega_{0}^{2}}{2} q^{2}, \quad \omega_{0}=\text { const. }
$$

Here, $H_{k}(q), k=0,1,2, \ldots$ denote the standard Hermite polynomials generated by the expression $\exp \left(2 q \xi-\xi^{2}\right)=\sum_{k=0}^{\infty} \frac{\xi^{k}}{k !} H_{k}(q)$ and $N_{k}=\left(2^{k} k !\right)^{-1 / 2}\left(\Omega_{0} / \pi\right)^{1 / 4}$ are the normalization constants, $\left(\Omega_{0}=\omega_{0} / \hbar\right)$. Therefore, the time-evolved state is

$$
\Psi(q, t)=\sum_{k=0}^{\infty}\left\langle\psi, \varphi_{k}\right\rangle \widehat{\mathcal{U}}\left(t, t_{0}\right) \varphi_{k}(q)=\sum_{k=0}^{\infty}\left\langle\psi, \varphi_{k}\right\rangle \Psi_{k}(q, t),
$$

where

$$
\begin{aligned}
\Psi_{k}(q, t)= & N_{k} \sqrt{R(t)} \times \exp \left(i\left(k+\frac{1}{2}\right) \arctan \left(\Omega_{0} g(t)\right)\right) \\
& \times \exp \left(i\left(\frac{\mu(t) \dot{x}(t)}{2 \hbar x(t)}-\frac{\Omega_{0}^{2}}{2} g(t) R^{2}(t)\right) q^{2}\right) \times \exp \left(-\frac{\Omega_{0}}{2} R^{2}(t) q^{2}\right) \times H_{k}\left(\sqrt{\Omega_{0}} R(t) q\right),
\end{aligned}
$$

and

$$
R(t)=\left(\frac{x_{0}^{2}}{x^{2}(t)+\left(\Omega_{0} x(t) g(t)\right)^{2}}\right)^{\frac{1}{2}} .
$$

The corresponding probability densities $\rho_{k}(q, t)=\left|\Psi_{k}(q, t)\right|^{2}$ are,

$$
\rho_{k}(q, t)=\frac{1}{2^{k} k ! \sqrt{\pi}} \times \sqrt{\Omega_{0}} R(t) \times \exp \left(-\left(\sqrt{\Omega_{0}} R(t) q\right)^{2}\right) \times H_{k}^{2}\left(\sqrt{\Omega_{0}} R(t) q\right), \quad k=0,1,2, \ldots
$$




\section{A. Madelung representation}

Madelung representation of the complex-valued wave function

$$
\Psi(q, t)=\sqrt{\rho(q, t)} \exp \left(\frac{i}{\hbar} S(q, t)\right)=\exp \left(\frac{1}{2} \ln \rho(q, t)+\frac{i}{\hbar} S(q, t)\right),
$$

where $\rho$ is the probability density and $S$ is the action, both being real-valued functions, decomposes the Schrödinger equation (1) into a system of nonlinear coupled partial differential equations:

$$
\left\{\begin{array}{l}
\frac{\partial S}{\partial t}+\frac{1}{2 \mu(t)}\left(\frac{\partial S}{\partial q}\right)^{2}+\frac{\mu(t) \omega^{2}(t)}{2} q^{2}=\frac{\hbar^{2}}{2 \mu(t)}\left[\frac{1}{\sqrt{\rho}} \frac{\partial^{2} \sqrt{\rho}}{\partial q^{2}}\right], \\
\frac{\partial \rho}{\partial t}+\frac{\partial}{\partial q}\left[\rho \frac{1}{\mu(t)} \frac{\partial S}{\partial q}\right]=0 .
\end{array}\right.
$$

The first equation may be viewed as a generalization of the usual Hamilton-Jacobi equation. The term with explicit $\hbar$ dependence is the quantum potential $Q(q, t)=-\frac{\hbar^{2}}{2 \mu(t)}\left[\frac{1}{\sqrt{\rho}} \frac{\partial^{2} \sqrt{\rho}}{\partial q^{2}}\right]$, encoding the quantum aspects of the theory. When $\hbar \rightarrow 0$, the equation becomes Hamilton-Jacobi equation for a non-relativistic particle with time-dependent mass. The second equation is a continuity equation expressing the conservation of probability density.

General IVP: Using the relation (12), one can see that the system (13), with general initial conditions

$$
S\left(q, t_{0}\right)=\tilde{S}(q), \quad \rho\left(q, t_{0}\right)=\tilde{\rho}(q),
$$

$\tilde{S}(q), \tilde{\rho}(q)$ being real-valued functions has formal solution

$$
S(q, t)=-i \hbar \ln \left(\frac{\Psi(q, t)}{|\Psi(q, t)|}\right), \quad \rho(q, t)=|\Psi(q, t)|^{2},
$$

where $\Psi(q, t)$ is a solution of the Schrödinger equation (1) with initial condition

$$
\psi(q)=\sqrt{\tilde{\rho}(q)} \exp \left(\frac{i}{\hbar} \tilde{S}(q)\right)
$$

Clearly, the explicit form of the solutions $S(q, t), \rho(q, t)$ depends on the properties of the initial functions $\tilde{\rho}(q)$ and $\tilde{S}(q)$. In this work, as in the following special IVP, we shall consider initial functions $\tilde{\rho}(q), \tilde{S}(q)$ so that in (16) one has $\psi(q) \in L_{2}(R)$. Then, $\Psi(q, t)$ can be found using (9) and one can obtain explicitly the solution (15) of the system (13) with initial conditions (14). Note also that, in general the action $S(q, t)=-i \hbar \ln (\Psi /|\Psi|)+2 \pi n \hbar, n=0, \pm 1, \pm 2, \ldots$ is multivalued, but fixing the initial condition $S\left(q, t_{0}\right)=\tilde{S}(q)$ leads to a single-valued solution of the IVP.

Special IVP: According to the above general discussion, we find that the system (13) with specific initial conditions

$$
S_{k}\left(q, t_{0}\right)=0, \quad \rho_{k}\left(q, t_{0}\right)=\varphi_{k}^{2}(q)=N_{k}^{2} \exp \left(-\left(\sqrt{\Omega_{0}} q\right)^{2}\right) H_{k}^{2}\left(\sqrt{\Omega_{0}} q\right), \quad k=0,1,2, \ldots
$$

has exact solutions

$$
\begin{gathered}
S_{k}(q, t)=-i \hbar \ln \left(\frac{\Psi_{k}(q, t)}{\left|\Psi_{k}(q, t)\right|}\right)=\frac{1}{2}\left(\mu(t) \frac{\dot{x}(t)}{x(t)}-\hbar \Omega_{0}^{2} g(t) R^{2}(t)\right) q^{2}+\hbar\left(k+\frac{1}{2}\right) \arctan \left(\Omega_{0} g(t)\right), \\
\rho_{k}(q, t)=\left|\Psi_{k}(q, t)\right|^{2}=\frac{1}{2^{k} k ! \sqrt{\pi}} \times \sqrt{\Omega_{0}} R(t) \times \exp \left(-\left(\sqrt{\Omega_{0}} R(t) q\right)^{2}\right) \times H_{k}^{2}\left(\sqrt{\Omega_{0}} R(t) q\right),
\end{gathered}
$$

where $\Psi_{k}(q, t)$ is given by $(9)$ and $\rho_{k}(q, t)$ is same as (11). 


\section{B. Madelung hydrodynamic equations}

Introducing classical velocity, $v(q, t)=\frac{1}{\mu(t)} \frac{\partial S}{\partial q}$, the system (13) transforms to Madelung fluid coupled system of equations with time variable coefficients :

$$
\left\{\begin{array}{l}
\frac{\partial v}{\partial t}+\frac{\dot{\mu}(t)}{\mu(t)} v+v \frac{\partial v}{\partial q}=-\frac{1}{\mu(t)} \frac{\partial}{\partial q}\left[\frac{-\hbar^{2}}{2 \mu(t)}\left(\frac{1}{\sqrt{\rho}} \frac{\partial^{2} \sqrt{\rho}}{\partial q^{2}}\right)+\frac{\mu(t) \omega^{2}(t)}{2} q^{2}\right], \\
\frac{\partial \rho}{\partial t}+\frac{\partial}{\partial q}[\rho v]=0,
\end{array}\right.
$$

where $\rho(q, t)$ is the density and $v(q, t)$ is the velocity field of the one-dimensional fluid. The hydrodynamical interpretation of the Schrödinger equation was first introduced by Madelung (Ref. 2). One can see also (Ref. 3). As well known, the only formal difference between the Madelung (quantum) fluid equations and the Euler (classical) equations of hydrodynamics is the quantum potential term containing the Planck constant $\hbar$.

General IVP: The system of fluid equation (17), with general initial conditions

$$
v\left(q, t_{0}\right)=\tilde{v}(q), \quad \rho\left(q, t_{0}\right)=\tilde{\rho}(q),
$$

$\tilde{v}(q), \tilde{\rho}(q)$ being real-valued functions, has formal solution

$$
v(q, t)=-\frac{i \hbar}{\mu(t)} \frac{\partial}{\partial q} \ln \left(\frac{\Psi(q, t)}{|\Psi(q, t)|}\right), \quad \rho(q, t)=|\Psi(q, t)|^{2},
$$

where $\Psi(q, t)$ is solution of the Schrödinger equation (1) with the initial condition

$$
\psi(q)=\sqrt{\tilde{\rho}(q)} \exp \left(\frac{i}{\hbar} \mu\left(t_{0}\right) \int^{q} \tilde{v}(\xi) d \xi\right) .
$$

Special IVP: The system (17) with specific initial conditions

$$
v_{k}\left(q, t_{0}\right)=0, \quad \rho_{k}\left(q, t_{0}\right)=\varphi_{k}^{2}(q), \quad k=0,1,2, \ldots,
$$

has exact solutions

$$
v_{k}(q, t)=-\frac{i \hbar}{\mu(t)} \frac{\partial}{\partial q} \ln \left(\frac{\Psi_{k}(q, t)}{\left|\Psi_{k}(q, t)\right|}\right)=\left(\frac{\dot{x}(t)}{x(t)}-\hbar \Omega_{0}^{2} \frac{g(t) R^{2}(t)}{\mu(t)}\right) q,
$$

and $\rho_{k}(q, t)$ as found before.

Note that, solving the equation $d q / d t=v(q, t)$ gives $q(t)=c / R(t)=$ $c \sqrt{x^{2}(t)+\left(\Omega_{0} g(t) x(t)\right)^{2}}$, where the integration constant $c$ is fixed by the initial position $q\left(t_{0}\right)=q_{0}$. These can be seen as "quantum streamlines," analogue to classical trajectories.

\section{POTENTIAL SCHRÖDINGER-BURGERS EQUATION}

Writing the wave function in the form $\Psi(q, t)=\exp \left(\frac{i}{\hbar} \mu(t) F(q, t)\right)$, where $F(q, t)$ is a complex potential, the linear Schrödinger equation (1) transforms to the nonlinear potential SchrödingerBurgers equation:

$$
\frac{\partial F}{\partial t}+\frac{\dot{\mu}(t)}{\mu(t)} F+\frac{1}{2}\left(\frac{\partial F}{\partial q}\right)^{2}+\frac{\omega^{2}(t)}{2} q^{2}=\frac{i \hbar}{2 \mu(t)} \frac{\partial^{2} F}{\partial q^{2}} .
$$

General IVP: The IVP for the potential Schrödinger-Burgers equation

$$
\left\{\begin{array}{l}
\frac{\partial F}{\partial t}+\frac{\dot{\mu}(t)}{\mu(t)} F+\frac{1}{2}\left(\frac{\partial F}{\partial q}\right)^{2}+\frac{\omega^{2}(t)}{2} q^{2}=\frac{i \hbar}{2 \mu(t)} \frac{\partial^{2} F}{\partial q^{2}}, \\
F\left(q, t_{0}\right)=\widetilde{F}(q),
\end{array}\right.
$$

has a formal solution given by

$$
F(q, t)=-\frac{i \hbar}{\mu(t)}(\ln \Psi(q, t)),
$$


where $\Psi(q, t)$ is a solution of the IVP for the Schrödinger equation

$$
\left\{\begin{array}{l}
i \hbar \frac{\partial \Psi}{\partial t}=-\frac{\hbar^{2}}{2 \mu(t)} \frac{\partial^{2} \Psi}{\partial q^{2}}+\frac{\mu(t) \omega^{2}(t)}{2} q^{2} \Psi \\
\Psi\left(q, t_{0}\right)=\psi(q)=\exp \left(\frac{i}{\hbar} \mu\left(t_{0}\right) \widetilde{F}(q)\right)
\end{array}\right.
$$

If the initial condition $\widetilde{F}(q)$ is such that $\psi(q) \in L_{2}(R)$, then by (8), the solution of the initial value problem (22) is

$$
F(q, t)=-\frac{i \hbar}{\mu(t)} \ln \sum_{k=0}^{\infty}\left\langle\psi, \varphi_{k}\right\rangle \Psi_{k}(q, t) .
$$

In particular, we have the following IVP with specific initial conditions.

\section{Special IVP:}

$$
\left\{\begin{array}{l}
\frac{\partial F}{\partial t}+\frac{\dot{\mu}(t)}{\mu(t)} F+\frac{1}{2}\left(\frac{\partial F}{\partial q}\right)^{2}+\frac{\omega^{2}(t)}{2} q^{2}=\frac{i \hbar}{2 \mu(t)} \frac{\partial^{2} F}{\partial q^{2}} \\
F_{k}\left(q, t_{0}\right)=-\frac{i \hbar}{\mu\left(t_{0}\right)}\left(\ln \varphi_{k}(q)\right)=\frac{i \hbar}{\mu\left(t_{0}\right)}\left(\frac{\Omega_{0}}{2} q^{2}-\ln \left(N_{k} H_{k}\left(\sqrt{\Omega_{0}} q\right)\right)\right), k=0,1,2, \ldots
\end{array}\right.
$$

has exact solutions

$$
\begin{aligned}
F_{k}(q, t)= & {\left[\frac{1}{2}\left(\frac{\dot{x}(t)}{x(t)}-\hbar \Omega_{0}^{2} \frac{g(t)}{\mu(t)} R^{2}(t)\right) q^{2}+\frac{\hbar}{\mu(t)}\left(k+\frac{1}{2}\right) \arctan \left(\Omega_{0} g(t)\right)\right] } \\
& +\frac{i \hbar}{\mu(t)}\left[\frac{\Omega_{0}}{2} R^{2}(t) q^{2}-\ln \left(N_{k} \sqrt{R(t)} H_{k}\left(\sqrt{\Omega_{0}} R(t) q\right)\right)\right]
\end{aligned}
$$

Now, using the Madelung representation (12) and relation (23), one can write

$$
F(q, t)=\mathrm{F}_{1}(q, t)+i \mathrm{~F}_{2}(q, t)=\frac{1}{\mu(t)} S(q, t)-\frac{i \hbar}{2 \mu(t)} \ln \rho(q, t),
$$

where $F_{1}$ represents the velocity potential and $F_{2}$ the stream function of the fluid, $\left(F_{1}, F_{2}\right.$ being real valued). Accordingly, the real and imaginary parts of the potential Schrödinger-Burgers equation (21) become

$$
\left\{\begin{array}{l}
\frac{\partial \mathrm{F}_{1}}{\partial t}+\frac{\dot{\mu}}{\mu} \mathrm{F}_{1}+\frac{1}{2}\left(\left(\frac{\partial \mathrm{F}_{1}}{\partial q}\right)^{2}-\left(\frac{\partial \mathrm{F}_{2}}{\partial q}\right)^{2}\right)+\frac{\omega^{2}(t)}{2} q^{2}=-\frac{\hbar}{2 \mu} \frac{\partial^{2} \mathrm{~F}_{2}}{\partial q^{2}} \\
\frac{\partial \mathrm{F}_{2}}{\partial t}+\frac{\dot{\mu}}{\mu} \mathrm{F}_{2}+\frac{\partial \mathrm{F}_{1}}{\partial q} \frac{\partial \mathrm{F}_{2}}{\partial q}=\frac{\hbar}{2 \mu} \frac{\partial^{2} \mathrm{~F}_{1}}{\partial q^{2}} .
\end{array}\right.
$$

General IVP: Using the relations (28) and (15), one can see that the nonlinear system (29) with general initial conditions

$$
\mathrm{F}_{1}\left(q, t_{0}\right)=\widetilde{\mathrm{F}}_{1}(q), \quad \mathrm{F}_{2}\left(q, t_{0}\right)=\widetilde{\mathrm{F}}_{2}(q),
$$

and $\widetilde{\mathrm{F}}_{1}(q), \widetilde{\mathrm{F}}_{2}(q)$ real-valued functions, has formal solution

$$
\mathrm{F}_{1}(q, t)=-\frac{i \hbar}{\mu(t)} \ln \left(\frac{\Psi(q, t)}{|\Psi(q, t)|}\right), \quad \mathrm{F}_{2}(q, t)=-\frac{\hbar}{\mu(t)} \ln (|\Psi(q, t)|),
$$

where $\Psi(q, t)$ is solution of the Schrödinger equation (1) with initial condition

$$
\Psi\left(q, t_{0}\right)=\psi(q)=\exp \left(\frac{i}{\hbar} \mu\left(t_{0}\right) \widetilde{\mathrm{F}}_{1}(q)\right) \times \exp \left(-\frac{1}{\hbar} \mu\left(t_{0}\right) \widetilde{\mathrm{F}}_{2}(q)\right) .
$$

Special IVP: Solutions of the system (29) with specific initial conditions

$$
\mathrm{F}_{1, k}\left(q, t_{0}\right)=0, \quad \mathrm{~F}_{2, k}\left(q, t_{0}\right)=\frac{\hbar}{\mu\left(t_{0}\right)}\left(\frac{\Omega_{0}}{2} q^{2}-\ln \left(N_{k} H_{k}\left(\sqrt{\Omega_{0}} q\right)\right), \quad k=0,1,2, \ldots\right.
$$


are clearly the real and imaginary parts of (27), that is

$$
\begin{gathered}
\mathrm{F}_{1, k}(q, t)=\left[\frac{1}{2}\left(\frac{\dot{x}(t)}{x(t)}-\hbar \Omega_{0}^{2} \frac{g(t)}{\mu(t)} R^{2}(t)\right) q^{2}+\frac{\hbar}{\mu(t)}\left(k+\frac{1}{2}\right) \arctan \left(\Omega_{0} g(t)\right)\right] \\
\mathrm{F}_{2, k}(q, t)=\frac{\hbar}{\mu(t)}\left[\frac{\Omega_{0}}{2} R^{2}(t) q^{2}-\ln \left(N_{k} \sqrt{R(t)} H_{k}\left(\sqrt{\Omega_{0}} R(t) q\right)\right)\right] .
\end{gathered}
$$

\section{SCHRÖDINGER-BURGERS EQUATION}

Representation of the wave function in the form

$$
\Psi(q, t)=\exp \left(\frac{i}{h} \mu(t) \int^{q} V(\xi, t) d \xi\right),
$$

where $V(q, t)$ is a complex velocity, transforms the Schrödinger equation (1) to the nonlinear Schrödinger-Burgers equation with time-dependent coefficients

$$
\frac{\partial V}{\partial t}+\frac{\dot{\mu}(t)}{\mu(t)} V+V \frac{\partial V}{\partial q}+\omega^{2}(t) q=\frac{i \hbar}{2 \mu(t)} \frac{\partial^{2} V}{\partial q^{2}} .
$$

This transformation, as before, allows us to obtain solutions of the nonlinear problem in terms of the corresponding linear one, as follows.

General IVP: The IVP for the Schrödinger-Burgers equation

$$
\left\{\begin{array}{l}
\frac{\partial V}{\partial t}+\frac{\dot{\mu}(t)}{\mu(t)} V+V \frac{\partial V}{\partial q}+\omega^{2}(t) q=\frac{i \hbar}{2 \mu(t)} \frac{\partial^{2} V}{\partial q^{2}} \\
V\left(q, t_{0}\right)=\widetilde{V}(q)
\end{array}\right.
$$

has formal solution given by the complex Cole-Hopf transformation

$$
V(q, t)=-\frac{i \hbar}{\mu(t)} \frac{\partial}{\partial q}(\ln \Psi(q, t)),
$$

where $\Psi(q, t)$ is solution of the IVP for the Schrödinger equation

$$
\left\{\begin{array}{l}
i \hbar \frac{\partial \Psi}{\partial t}=-\frac{\hbar^{2}}{2 \mu(t)} \frac{\partial^{2} \Psi}{\partial q^{2}}+\frac{\mu(t) \omega^{2}(t)}{2} q^{2} \Psi \\
\Psi\left(q, t_{0}\right)=\psi(q)=\exp \left(\frac{i}{\hbar} \mu\left(t_{0}\right) \int^{q} \widetilde{V}(\xi) d \xi\right) .
\end{array}\right.
$$

If the initial condition $\widetilde{V}(q)$ is such that $\psi(q) \in L_{2}(R)$, then by (8), the solution of (32) is

$$
V(q, t)=\frac{-i \hbar \sum_{k=0}^{\infty}\left\langle\psi, \varphi_{k}\right\rangle \partial_{q} \Psi_{k}(q, t)}{\mu(t) \sum_{k=0}^{\infty}\left\langle\psi, \varphi_{k}\right\rangle \Psi_{k}(q, t)},
$$

where $\partial_{q}$ denotes differentiation with respect to $q$. A particular case is the following IVP with specific initial conditions.

Special IVP: The problem

$$
\left\{\begin{array}{l}
\frac{\partial V}{\partial t}+\frac{\dot{\mu}(t)}{\mu(t)} V+V \frac{\partial V}{\partial q}+\omega^{2}(t) q=\frac{i \hbar}{2 \mu(t)} \frac{\partial^{2} V}{\partial q^{2}}, \\
V_{k}\left(q, t_{0}\right)=-\frac{i \hbar}{\mu\left(t_{0}\right)} \frac{d}{d q}\left(\ln \varphi_{k}(q)\right)=\frac{i \hbar}{\mu\left(t_{0}\right)}\left[\Omega_{0} q-\frac{\partial_{q} H_{k}\left(\sqrt{\Omega_{0}} q\right)}{H_{k}\left(\sqrt{\Omega_{0}} q\right)}\right], k=0,1,2, \ldots
\end{array}\right.
$$

has exact solution of the form

$$
V_{k}(q, t)=\left[\frac{\dot{x}(t)}{x(t)}-\hbar \Omega_{0}^{2} \frac{g(t)}{\mu(t)} R^{2}(t)\right] q+\frac{i \hbar}{\mu(t)}\left[\Omega_{0} R^{2}(t) q-\frac{\partial_{q} H_{k}\left(\sqrt{\Omega_{0}} R(t) q\right)}{H_{k}\left(\sqrt{\Omega_{0}} R(t) q\right)}\right], \quad k=0,1,2, \ldots
$$


Using the Madelung representation (12) and the complex Cole-Hopf transformation (33), one can write the complex velocity function in the form

$$
V(q, t)=v(q, t)+i u(q, t)=\frac{1}{\mu(t)} \frac{\partial S}{\partial q}-\frac{i \hbar}{2 \mu(t)} \frac{\partial}{\partial q}(\ln \rho),
$$

where $v, u$ are real valued, $v$ represents the classical velocity and $u$ the quantum velocity. This splits the Schrödinger-Burgers equation into real and imaginary parts, respectively:

$$
\left\{\begin{array}{l}
\frac{\partial v}{\partial t}+\frac{\dot{\mu}(t)}{\mu(t)} v+v \frac{\partial v}{\partial q}+\omega^{2}(t) q=\frac{-\hbar}{2 \mu(t)} \frac{\partial^{2} u}{\partial q^{2}}+u \frac{\partial u}{\partial q}, \\
\frac{\partial u}{\partial t}+\frac{\dot{\mu}(t)}{\mu(t)} u+u \frac{\partial v}{\partial q}+v \frac{\partial u}{\partial q}=\frac{\hbar}{2 \mu(t)} \frac{\partial^{2} v}{\partial q^{2}} .
\end{array}\right.
$$

General IVP: Using relations (37) and (15), we find that the system of nonlinear coupled equations (38) with general initial conditions

$$
v\left(q, t_{0}\right)=\tilde{v}(q), \quad u\left(q, t_{0}\right)=\tilde{u}(q)
$$

has formal solution

$$
v(q, t)=-\frac{i \hbar}{\mu(t)} \frac{\partial}{\partial q} \ln \left(\frac{\Psi(q, t)}{|\Psi(q, t)|}\right), \quad u(q, t)=-\frac{\hbar}{\mu(t)} \frac{\partial}{\partial q} \ln (|\Psi(q, t)|),
$$

where $\Psi(q, t)$ is a solution of the Schrödinger equation (1) with general initial condition

$$
\Psi\left(q, t_{0}\right)=\exp \left(\frac{i}{\hbar} \mu\left(t_{0}\right) \int^{q} \tilde{v}(\xi) d \xi\right) \times \exp \left(-\frac{1}{\hbar} \mu\left(t_{0}\right) \int^{q} \tilde{u}(\xi) d \xi\right) .
$$

Special IVP: System (38) with specific initial conditions

$$
v_{k}\left(q, t_{0}\right)=0, \quad u_{k}\left(q, t_{0}\right)=\frac{\hbar}{\mu\left(t_{0}\right)}\left[\Omega_{0} q-\frac{\partial_{q} H_{k}\left(\sqrt{\Omega_{0}} q\right)}{H_{k}\left(\sqrt{\Omega_{0}} q\right)}\right], \quad k=0,1,2, \ldots
$$

has exact solutions

$$
v_{k}(q, t)=\left[\frac{\dot{x}(t)}{x(t)}-\hbar \Omega_{0}^{2} \frac{g(t)}{\mu(t)} R^{2}(t)\right] q, \quad u_{k}(q, t)=\frac{\hbar}{\mu(t)}\left[\Omega_{0} R^{2}(t) q-\frac{\partial_{q} H_{k}\left(\sqrt{\Omega_{0}} R(t) q\right)}{H_{k}\left(\sqrt{\Omega_{0}} R(t) q\right)}\right] .
$$

Clearly, the quantity $\left|V_{k}(q, t)\right|^{2}=v_{k}^{2}(q, t)+u_{k}^{2}(q, t)$ which is analog of kinetic energy can be also explicitly computed. In addition, $\left|V_{k}(q, t)\right|$ can be interpreted also as an amplitude of envelope of the modulated wave.

Motion of zeros and poles: Due to the complex Cole-Hopf transformation (33), the zeros of the wave function $\Psi(q, t)$ (and $\rho(q, t))$ for the Schrödinger equation become poles of the solution $V(q, t)$ (and $|V(q, t)|^{2}$ ) for the nonlinear Schrödinger-Burgers equation (32). In particular, we note that $\Psi_{k}(q, t)$ given by (9) has zeros at points where $H_{k}\left(\sqrt{\Omega_{0}} R(t) q\right)=0$, and these points are pole singularities for $V_{k}(q, t)$ given by (36). Thus, denoting by $\tau_{k}^{(l)}, l=1,2, \ldots k$, the zeros of the Hermite polynomial $H_{k}(\xi)$, i.e., $H_{k}\left(\tau_{k}^{(l)}\right)=0$, we obtain that for each $k=1,2,3, \ldots$, the motion of the zeros and poles is given by

$$
q_{k}^{(l)}(t)=\frac{\tau_{k}^{(l)}}{\sqrt{\Omega_{0}} R(t)}=\frac{\tau_{k}^{(l)}}{\left|x_{0}\right| \sqrt{\Omega_{0}}} \sqrt{x^{2}(t)+\left(\Omega_{0} g(t) x(t)\right)^{2}}, \quad l=1,2, \ldots, k .
$$

General analysis of the system describing the dynamics of the zeros and poles will be presented in later work. However, for some particular cases the motion of zeros and poles is discussed and illustrated in Sec. V. 


\section{EXACTLY SOLVABLE MODELS}

\section{A. Madelung representation of standard quantum harmonic oscillator}

The subject of this work is to study nonlinear models with time-dependent parameters, but for comparative reasons we briefly mention about the case when the parameters are constant, that is $\mu(t)=1$ and $\omega^{2}(t)=\omega_{0}^{2}$. Thus, we start with IVP for the standard quantum harmonic oscillator

$$
\left\{\begin{array}{l}
i \hbar \frac{\partial \Psi}{\partial t}=-\frac{\hbar^{2}}{2} \frac{\partial^{2} \Psi}{\partial q^{2}}+\frac{\omega_{0}^{2}}{2} q^{2} \Psi \\
\Psi\left(q, t_{0}\right)=\varphi_{k}(q), \quad k=0,1,2, \ldots
\end{array}\right.
$$

which has well known solutions

$$
\Psi_{k}(q, t)=e^{-\frac{i}{\hbar}\left(k+\frac{1}{2}\right)\left(t-t_{0}\right)} \varphi_{k}(q), \quad k=0,1,2, \ldots,
$$

in terms of $\varphi_{k}(q)$ given by (6). To find these solutions, one can use also formula (9) with $x(t)=$ $x_{0} \cos \left[\omega_{0}\left(t-t_{0}\right)\right], g(t)=-\frac{\hbar}{\omega_{0}} \tan \left[\omega_{0}\left(t-t_{0}\right)\right]$, and $R(t)=1$. It follows that:

$a$. The system for generalized Hamilton-Jacobi and continuity equations with specific initial conditions, that is

$$
\left\{\begin{array}{l}
\frac{\partial S}{\partial t}+\frac{1}{2}\left(\frac{\partial S}{\partial q}\right)^{2}+\frac{\omega_{0}^{2}}{2} q^{2}=\frac{\hbar^{2}}{2}\left[\frac{1}{\sqrt{\rho}} \frac{\partial^{2} \sqrt{\rho}}{\partial q^{2}}\right], \\
\frac{\partial \rho}{\partial t}+\frac{\partial}{\partial q}\left[\rho \frac{\partial S}{\partial q}\right]=0, \\
S_{k}\left(q, t_{0}\right)=0, \quad \rho_{k}\left(q, t_{0}\right)=\varphi_{k}^{2}(q), \quad k=0,1,2, \ldots,
\end{array}\right.
$$

has solutions

$$
S_{k}(q, t)=-\hbar \omega_{0}\left(k+\frac{1}{2}\right)\left(t-t_{0}\right), \quad \rho_{k}(q, t)=\varphi_{k}^{2}(q), \quad k=0,1,2, \ldots
$$

$b$. The IVP for the hydrodynamic equations

$$
\left\{\begin{array}{l}
\frac{\partial v}{\partial t}+v \frac{\partial v}{\partial q}=-\frac{\partial}{\partial q}\left[\frac{-\hbar^{2}}{2}\left(\frac{1}{\sqrt{\rho}} \frac{\partial^{2} \sqrt{\rho}}{\partial q^{2}}\right)+\frac{\omega_{0}^{2}}{2} q^{2}\right] \\
\frac{\partial \rho}{\partial t}+\frac{\partial}{\partial q}[\rho v]=0 \\
v_{k}\left(q, t_{0}\right)=0, \quad \rho_{k}\left(q, t_{0}\right)=\varphi_{k}^{2}(q), \quad k=0,1,2, \ldots
\end{array}\right.
$$

has solutions

$$
v_{k}(q, t)=0, \quad \rho_{k}(q, t)=\varphi_{k}^{2}(q), \quad k=0,1,2, \ldots
$$

$c$. The IVP for potential Schrödinger-Burgers equation

$$
\left\{\begin{array}{l}
\frac{\partial F}{\partial t}+\frac{1}{2}\left(\frac{\partial F}{\partial q}\right)^{2}+\frac{\omega_{0}^{2}}{2} q^{2}=\frac{i \hbar}{2} \frac{\partial^{2} F}{\partial q^{2}}, \\
F_{k}\left(q, t_{0}\right)=-i \hbar \ln \varphi_{k}(q), \quad k=0,1,2, \ldots
\end{array}\right.
$$

has solutions

$$
F_{k}(q, t)=-i \hbar \ln \Psi_{k}(q, t)=-\omega_{0}\left(k+\frac{1}{2}\right)\left(t-t_{0}\right)-i \hbar \ln \varphi_{k}(q), \quad k=0,1,2, \ldots
$$

$d$. The IVP for Schrödinger-Burgers equation

$$
\left\{\begin{array}{l}
\frac{\partial V}{\partial t}+V \frac{\partial V}{\partial q}+\omega_{0}^{2} q=\frac{i \hbar}{2} \frac{\partial^{2} V}{\partial q^{2}}, \\
V_{k}\left(q, t_{0}\right)=-i \hbar \frac{d}{d q}\left(\ln \varphi_{k}(q)\right)=i \hbar\left[\Omega_{0} q-\frac{\partial_{q} H_{k}\left(\sqrt{\Omega_{0}} q\right)}{H_{k}\left(\sqrt{\Omega_{0}} q\right)}\right], k=0,1,2, \ldots
\end{array}\right.
$$


has solutions

$$
V_{k}(q, t)=i \hbar\left[\Omega_{0} q-\frac{\partial_{q} H_{k}\left(\sqrt{\Omega_{0}} q\right)}{H_{k}\left(\sqrt{\Omega_{0}} q\right)}\right], k=0,1,2, \ldots,
$$

so that the classical and quantum velocities are

$$
v_{k}(q, t)=0, \quad u_{k}(q, t)=\hbar\left[\Omega_{0} q-\frac{\partial_{q} H_{k}\left(\sqrt{\Omega_{0}} q\right)}{H_{k}\left(\sqrt{\Omega_{0}} q\right)}\right] .
$$

Notice that, in this case, the zeros of the wave function $\Psi_{k}(q, t)$ and the poles of the solution $V_{k}(q, t)$ are the points $q_{k}^{(l)}=\frac{\tau_{k}^{(l)}}{\sqrt{\Omega_{0}}}, l=1,2, \ldots, k$, which are fixed in time.

\section{B. Madelung representation of Caldirola-Kanai oscillator}

The Caldirola-Kanai model, (Refs. 13 and 14), which is a one-dimensional system with an exponentially increasing mass, is the best known model of harmonic oscillator with time-dependent parameters. Here, using the general discussion in the previous parts, we obtain exact solutions of the nonlinear problems related with the Caldirola-Kanai oscillator:

$$
\left\{\begin{array}{l}
i \hbar \frac{\partial \Psi}{\partial t}=-\frac{\hbar^{2}}{2} e^{-\gamma t} \frac{\partial^{2} \Psi}{\partial q^{2}}+\frac{1}{2} \omega_{0}^{2} e^{\gamma t} q^{2} \Psi, \quad q \in R, t>0, \\
\Psi(q, 0)=\psi(q)
\end{array}\right.
$$

where $\mu(t)=e^{\gamma t}$ is the integrating factor, $\Gamma(t)=\gamma>0$ is the damping term, and $\omega^{2}(t)=\omega_{0}^{2}$ is a constant frequency. As stated in the beginning of Sec. II, solutions of the Cadirola-Kanai oscillator can be found in terms of the solution to the corresponding classical equation of motion:

$$
\ddot{x}+\gamma \dot{x}+\omega_{0}^{2} x=0, \quad x(0)=x_{0} \neq 0, \dot{x}(0)=0 .
$$

Clearly, according to the sign of $\Omega^{2}=\omega_{0}^{2}-\left(\gamma^{2} / 4\right)$ there are three different type of behaviorcritical damping, underdamping, and overdamping. For each type, nonlinear models discussed in Secs. II-IV are presented and exact solutions are found, as follows.

\section{Critical damping case, $\Omega^{2}=0$}

If $\Omega^{2}=\omega_{0}^{2}-\left(\gamma^{2} / 4\right)=0$, then the classical equation (42) has solution

$$
x_{1}(t)=x_{0} e^{-\frac{\gamma t}{2}}\left(1+\frac{\gamma}{2} t\right)
$$

and it follows from (5) and (10) that

$$
g_{1}(t)=\frac{-\hbar t}{1+\frac{\gamma}{2} t}, \quad R_{1}(t)=\left(\frac{e^{\gamma t}}{\left(1+\frac{\gamma}{2} t\right)^{2}+w_{0}^{2} t^{2}}\right)^{1 / 2} .
$$

Then, by (9), solutions of the Schrödinger equation (41) with initial conditions $\Psi(q, 0)=\varphi_{k}(q)$, are

$$
\begin{gathered}
\Psi_{k}(q, t)=N_{k}\left(\frac{e^{\gamma t}}{\left(1+\frac{\gamma}{2} t\right)^{2}+w_{0}^{2} t^{2}}\right)^{1 / 4} \times \exp \left(i\left(k+\frac{1}{2}\right) \arctan \left(\frac{-\omega_{0} t}{1+\frac{\gamma}{2} t}\right)\right) \\
\times \exp \left(-i \frac{\omega_{0}^{2}}{2 \hbar}\left(\frac{t e^{\gamma t}}{1+\frac{\gamma}{2} t}\right)\left(1-\frac{1}{\left(1+\frac{\gamma}{2} t\right)^{2}+w_{0}^{2} t^{2}}\right) q^{2}\right) \times \exp \left(-\frac{\omega_{0}}{2 \hbar}\left(\frac{e^{\gamma t}}{\left(1+\frac{\gamma}{2} t\right)^{2}+w_{0}^{2} t^{2}}\right) q^{2}\right) \\
\times H_{k}\left(\sqrt{\frac{\omega_{0}}{\hbar}}\left(\frac{e^{\gamma t}}{\left(1+\frac{\gamma}{2} t\right)^{2}+w_{0}^{2} t^{2}}\right)^{1 / 2} q\right), k=0,1,2, \ldots
\end{gathered}
$$


a. Hamilton-Jacobi-type representation of Caldirola-Kanai oscillator, $\Omega^{2}=0$. Madelung representation of the wave function decomposes the Schrödinger equation (41) into a system of nonlinear coupled partial differential equations:

$$
\left\{\begin{array}{l}
\frac{\partial S}{\partial t}+\frac{1}{2} e^{-\gamma t}\left(\frac{\partial S}{\partial q}\right)^{2}+\frac{\omega_{0}^{2}}{2} e^{\gamma t} q^{2}=\frac{\hbar^{2}}{2} e^{-\gamma t}\left[\frac{1}{\sqrt{\rho}} \frac{\partial^{2} \sqrt{\rho}}{\partial q^{2}}\right] \\
\frac{\partial \rho}{\partial t}+e^{-\gamma t} \frac{\partial}{\partial q}\left[\rho \frac{\partial S}{\partial q}\right]=0 .
\end{array}\right.
$$

This system of equations with specific initial conditions

$$
S_{k}(q, 0)=0, \quad \rho_{k}(q, 0)=N_{k}^{2} \exp \left(-\frac{\omega_{0}}{\hbar} q^{2}\right) H_{k}^{2}\left(\sqrt{\frac{\omega_{0}}{\hbar}} q\right), \quad k=0,1,2, \ldots,
$$

has exact solutions of the form

$$
\begin{gathered}
S_{k}(q, t)=\left(-\frac{\omega_{0}^{2}}{2}\left(\frac{t e^{\gamma t}}{1+\frac{\gamma}{2} t}\right)\left(1-\frac{1}{\left(1+\frac{\gamma}{2} t\right)^{2}+w_{0}^{2} t^{2}}\right) q^{2}\right)+\left(\hbar\left(k+\frac{1}{2}\right) \arctan \left(\frac{-\omega_{0} t}{1+\frac{\gamma}{2} t}\right)\right), \\
\rho_{k}(q, t)= \\
N_{k}^{2}\left(\frac{e^{\gamma t}}{\left(1+\frac{\gamma}{2} t\right)^{2}+w_{0}^{2} t^{2}}\right)^{1 / 2} \times \exp \left(-\frac{\omega_{0}}{\hbar}\left(\frac{e^{\gamma t}}{\left(1+\frac{\gamma}{2} t\right)^{2}+w_{0}^{2} t^{2}}\right) q^{2}\right) \\
\times H_{k}^{2}\left(\sqrt{\frac{\omega_{0}}{\hbar}}\left(\frac{e^{\gamma t}}{\left(1+\frac{\gamma}{2} t\right)^{2}+w_{0}^{2} t^{2}}\right)^{1 / 2} q\right) .
\end{gathered}
$$

b. Madelung hydrodynamic equations, $\Omega^{2}=0$. The system of hydrodynamic equations for the velocity and density of the fluid

$$
\left\{\begin{array}{l}
\frac{\partial v}{\partial t}+\gamma v+v \frac{\partial v}{\partial q}=-e^{-\gamma t} \frac{\partial}{\partial q}\left[\frac{-\hbar^{2}}{2} \frac{e^{-\gamma t}}{\sqrt{\rho}} \frac{\partial^{2} \sqrt{\rho}}{\partial q^{2}}+\frac{\omega_{0}^{2}}{2} e^{\gamma t} q^{2}\right] \\
\frac{\partial \rho}{\partial t}+\frac{\partial}{\partial q}[\rho v]=0,
\end{array}\right.
$$

with specific initial conditions

$$
v_{k}(q, 0)=0, \quad \rho_{k}(q, 0)=N_{k}^{2} \exp \left(-\frac{\omega_{0}}{\hbar} q^{2}\right) H_{k}^{2}\left(\sqrt{\frac{\omega_{0}}{\hbar}} q\right), \quad k=0,1,2, \ldots
$$

has solutions

$$
v_{k}(q, t)=\left(-\omega_{0}^{2}\left(\frac{t}{1+\frac{\gamma}{2} t}\right)\left(1-\frac{1}{\left(1+\frac{\gamma}{2} t\right)^{2}+w_{0}^{2} t^{2}}\right) q\right),
$$

and $\rho_{k}(q, t)$ given by $(45)$.

c. The potential Schrödinger-Burgers equation, $\Omega^{2}=0$. The IVP for potential SchrödingerBurgers equation

$$
\left\{\begin{array}{l}
\frac{\partial F}{\partial t}+\gamma F+\frac{1}{2}\left(\frac{\partial F}{\partial q}\right)^{2}+\frac{\omega_{0}^{2}}{2} q^{2}=\frac{i \hbar}{2} e^{-\gamma t}\left(\frac{\partial^{2} F}{\partial q^{2}}\right) \\
F_{k}(q, 0)=i\left(\frac{\omega_{0}}{2} q^{2}-\hbar \ln \left(N_{k} H_{k}\left(\sqrt{\frac{\omega_{0}}{\hbar}} q\right)\right)\right), \quad k=0,1,2, \ldots
\end{array}\right.
$$

has exact solutions of the form

$$
F_{k}(q, t)=\mathrm{F}_{1, k}(q, t)+i \mathrm{~F}_{2, k}(q, t),
$$




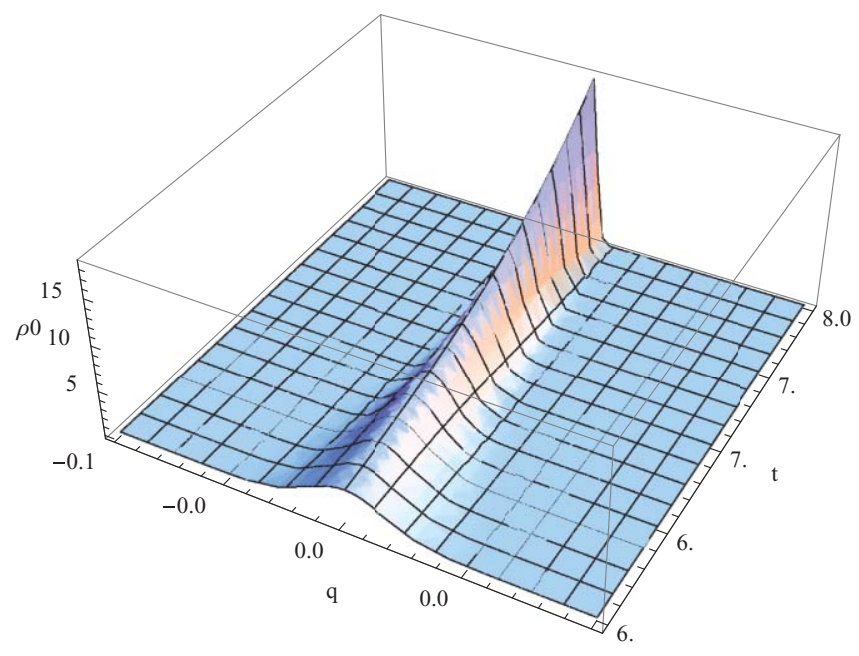

(a)

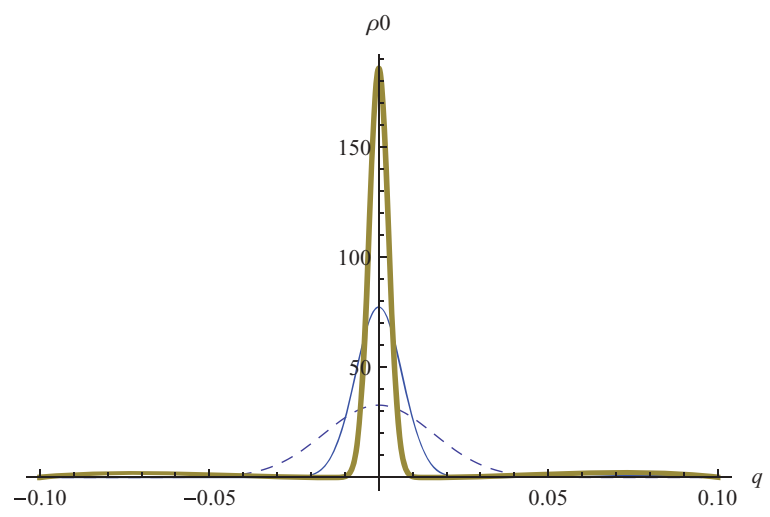

(b)

FIG. 1. Case $\Omega^{2}=0$. (a) Evolution of probability density $\rho_{0}(q, t)$. (b) Plot of $\rho_{0}(q, t)$ at times $t=6,7,8$, showing Dirac-delta behavior as $t \rightarrow \infty$.

where

$$
\begin{gathered}
\mathrm{F}_{1, k}=-\frac{\omega_{0}^{2}}{2}\left(\frac{t}{1+\frac{\gamma}{2} t}\right)\left(1-\frac{1}{\left(1+\frac{\gamma}{2} t\right)^{2}+w_{0}^{2} t^{2}}\right) q^{2}+\hbar e^{-\gamma t}\left(k+\frac{1}{2}\right) \arctan \left(\frac{-\omega_{0} t}{1+\frac{\gamma}{2}}\right), \\
\mathrm{F}_{2, k}=\frac{\omega_{0}}{2}\left(\frac{1}{\left(1+\frac{\gamma}{2} t\right)^{2}+w_{0}^{2} t^{2}}\right) q^{2}-\hbar e^{-\gamma t} \ln \left[N_{k}\left(\frac{e^{-(\gamma / 2) t}}{\left(1+\frac{\gamma}{2} t\right)^{2}+w_{0}^{2} t^{2}}\right)^{1 / 2}\right. \\
\left.\times H_{k}\left(\sqrt{\frac{\omega_{0}}{\hbar}\left(\frac{e^{\gamma t}}{\left(1+\frac{\gamma}{2} t\right)^{2}+w_{0}^{2} t^{2}}\right)} q\right)\right]
\end{gathered}
$$




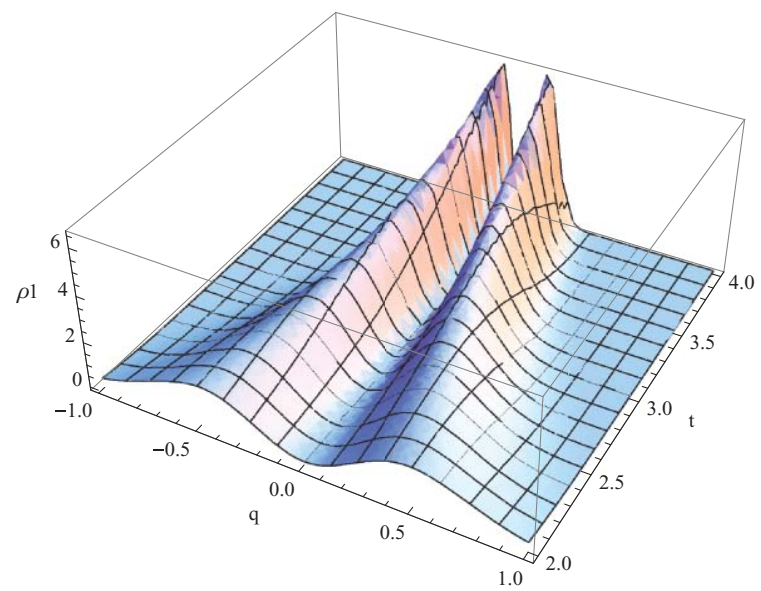

(a)

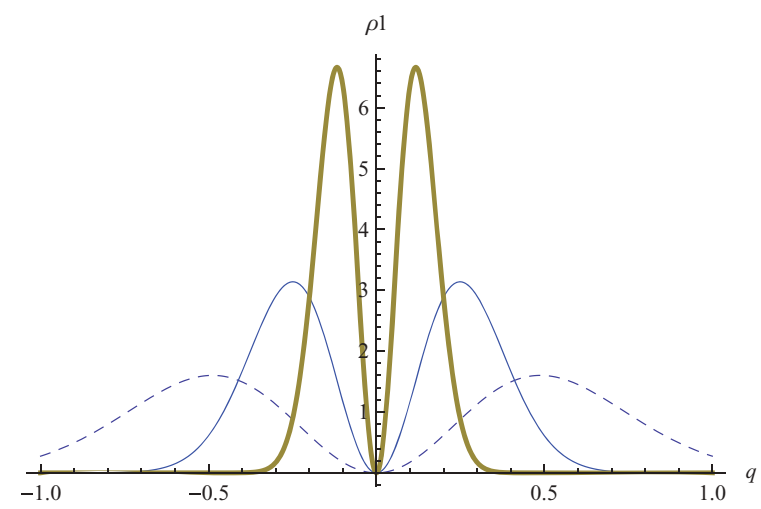

(b)

FIG. 2. Case $\Omega^{2}=0$. (a) Evolution of probability density $\rho_{1}(q, t)$. (b) Plot of $\rho_{1}(q, t)$ at times $t=2,3,4$.

The representation $F(q, t)=\mathrm{F}_{1}(q, t)+i \mathrm{~F}_{2}(q, t)$ decomposes the potential Schrödinger-Burgers equation (46) into the system:

$$
\left\{\begin{array}{l}
\frac{\partial \mathrm{F}_{1}}{\partial t}+\gamma \mathrm{F}_{1}+\frac{1}{2}\left(\left(\frac{\partial \mathrm{F}_{1}}{\partial q}\right)^{2}-\left(\frac{\partial \mathrm{F}_{2}}{\partial q}\right)^{2}\right)+\frac{\omega_{0}^{2}}{2} q^{2}=-\frac{\hbar e^{-\gamma t}}{2} \frac{\partial^{2} \mathrm{~F}_{2}}{\partial q^{2}} \\
\frac{\partial \mathrm{F}_{2}}{\partial t}+\gamma \mathrm{F}_{2}+\frac{\partial \mathrm{F}_{1}}{\partial q} \frac{\partial \mathrm{F}_{2}}{\partial q}=\frac{\hbar e^{-\gamma t}}{2} \frac{\partial^{2} \mathrm{~F}_{1}}{\partial q^{2}} .
\end{array}\right.
$$

Obviously, solutions of this system with specific initial conditions

$$
\mathrm{F}_{1, k}(q, 0)=0, \quad \mathrm{~F}_{2, k}(q, 0)=\left(\frac{\omega_{0}}{2} q^{2}-\hbar \ln \left(N_{k} H_{k}\left(\sqrt{\frac{\omega_{0}}{\hbar}} q\right)\right)\right)
$$

are the real and imaginary parts of $F_{k}$, that is $\mathrm{F}_{1, k}$ and $\mathrm{F}_{2, k}$ as found above.

$d$. The Schrödinger-Burgers equation, $\Omega^{2}=0$. The IVP for the nonlinear Schrödinger-Burgers equation

$$
\left\{\begin{array}{l}
\frac{\partial V}{\partial t}+\gamma V+V \frac{\partial V}{\partial q}+\omega_{0}^{2} q=i \hbar \frac{e^{-\gamma t}}{2} \frac{\partial^{2} V}{\partial q^{2}}, \\
V_{k}(q, 0)=i\left[\omega_{0} q-2 k \sqrt{\hbar \omega_{0}}\left(\frac{H_{k-1}\left(\sqrt{\frac{\omega_{0}}{\hbar}} q\right)}{H_{k}\left(\sqrt{\frac{\omega_{0}}{\hbar}} q\right)}\right)\right], k=0,1,2, \ldots,
\end{array}\right.
$$




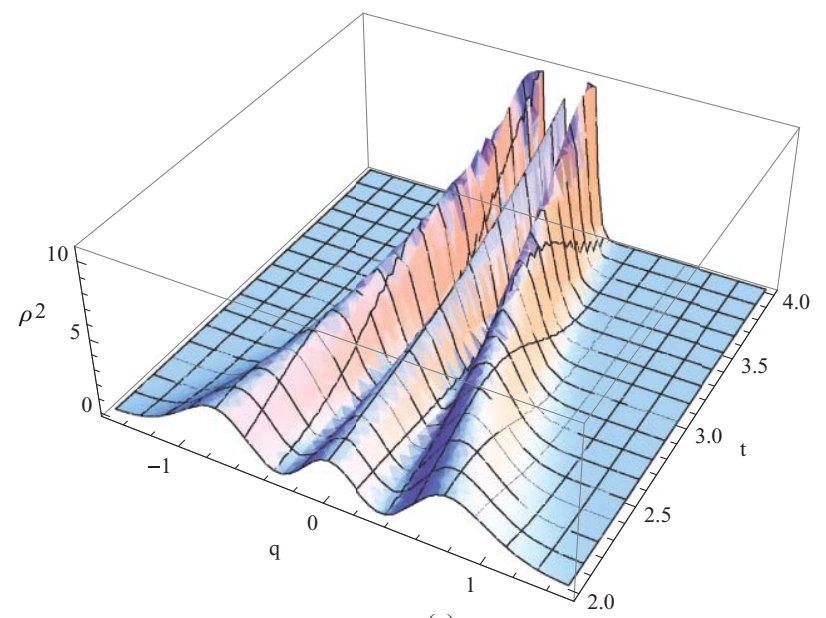

(a)

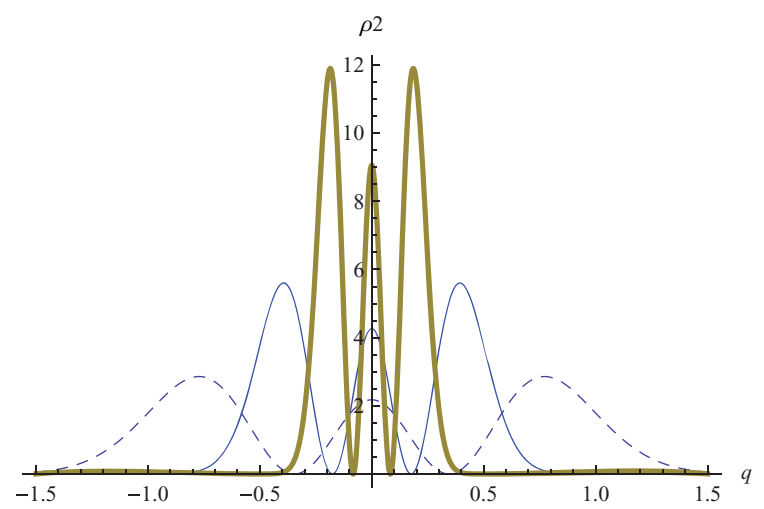

(b)

FIG. 3. Case $\Omega^{2}=0$. (a) Evolution of probability density $\rho_{2}(q, t)$. (b) Plot of $\rho_{2}(q, t)$ at times $t=2,3,4$.

has solutions

$$
\begin{array}{r}
V_{k}(q, t)=\left(-\omega_{0}^{2}\left(\frac{t}{1+\frac{\gamma}{2} t}\right)\left(1-\frac{1}{\left(1+\frac{\gamma}{2} t\right)^{2}+w_{0}^{2} t^{2}}\right) q\right) \\
+i\left[\left(\frac{\omega_{0}}{\left(1+\frac{\gamma}{2} t\right)^{2}+w_{0}^{2} t^{2}}\right) q-2 k \sqrt{\hbar \omega_{0}} e^{-\gamma t}\left(\frac{e^{\gamma t}}{\left(1+\frac{\gamma}{2} t\right)^{2}+w_{0}^{2} t^{2}}\right)\right. \\
\left.\times\left(\frac{H_{k-1}\left(\sqrt{\frac{\omega_{0}}{\hbar}\left(\frac{e^{\gamma t}}{\left(1+\frac{\gamma}{2} t\right)^{2}+w_{0}^{2} t^{2}}\right)} q\right)}{H_{k}\left(\sqrt{\left.\frac{\omega_{0}\left(\frac{e^{\gamma t}}{\hbar}\right.}{\left(1+\frac{\gamma}{2} t\right)^{2}+w_{0}^{2} t^{2}}\right)} q\right)}\right)\right] .
\end{array}
$$

We used that $\partial_{q} H_{k}(q)=2 k H_{k-1}(q)$ and $H_{-1}=0$.

The complex velocity function, written in the form $V(q, t)=v(q, t)+i u(q, t)$, where $v, u$ are real-valued functions, splits the Schrödinger-Burgers equation (47) into the system:

$$
\left\{\begin{array}{l}
\frac{\partial v}{\partial t}+\gamma v+v \frac{\partial v}{\partial q}-u \frac{\partial u}{\partial q}+\omega_{0}^{2} q=-\frac{\hbar}{2} e^{-\gamma t} \frac{\partial^{2} u}{\partial q^{2}} \\
\frac{\partial u}{\partial t}+\gamma u+u \frac{\partial v}{\partial q}+u \frac{\partial v}{\partial q}=\frac{\hbar}{2} e^{-\gamma t} \frac{\partial^{2} v}{\partial q^{2}}
\end{array}\right.
$$




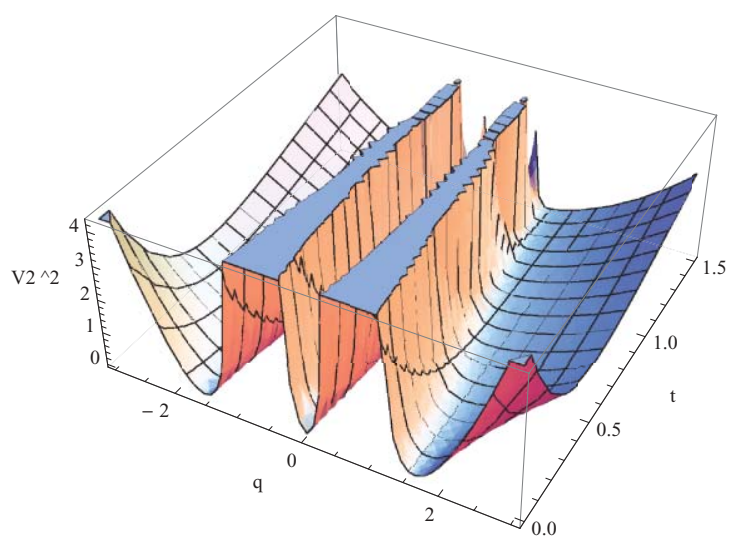

(a)

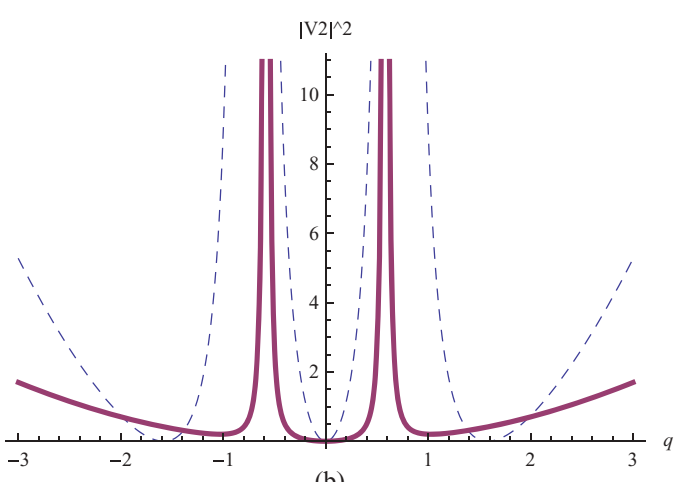

(b)

FIG. 4. Case $\Omega^{2}=0$. (a) Evolution of $\left|V_{2}(q, t)\right|^{2}$. (b) Behavior of $\left|V_{2}(q, t)\right|^{2}$ at times $t=0,1$.

This system with specific initial conditions

$$
v_{k}(q, 0)=0, \quad u_{k}(q, 0)=\omega_{0} q-2 k \sqrt{\hbar \omega_{0}}\left(\frac{H_{k-1}\left(\sqrt{\frac{\omega_{0}}{\hbar}} q\right)}{H_{k}\left(\sqrt{\frac{\omega_{0}}{\hbar}} q\right)}\right), \quad k=0,1,2, \ldots
$$

clearly, has solutions $v_{k}(q, t)$ and $u_{k}(q, t)$ which are respectively, the real and imaginary parts of $V_{k}(q, t)$ in expression (48).

e. Motion of zeros and poles, $\Omega^{2}=0$. From expression (43), we see that the solution $\Psi_{k}(q, t)$ (also $\rho_{k}(q, t)$ ) of the linear Schrödinger equation (41) has zeros at points where

$$
H_{k}\left(\sqrt{\frac{\omega_{0}}{\hbar}\left(\frac{e^{\gamma t}}{\left(1+\frac{\gamma}{2} t\right)^{2}+w_{0}^{2} t^{2}}\right)} q\right)=0,
$$

and these zeros are pole singularities of the solution $V_{k}(q, t)$ (also $\left.\left|V_{k}(q, t)\right|^{2}\right)$ for the nonlinear Schrödinger-Burgers equation (47). Therefore, for each fixed $k=0,1,2,3, \ldots$, the motion of the zeros and poles is described by the curves:

$$
q_{k}^{(l)}(t)=\tau_{k}^{(l)} \sqrt{\frac{\hbar}{\omega_{0}}} \times e^{-\frac{\gamma}{2} t} \times \sqrt{\left(1+\frac{\gamma}{2} t\right)^{2}+w_{0}^{2} t^{2}}, \quad l=1,2, \ldots k,
$$

where $\tau_{k}^{(l)}, l=1,2, \ldots k$, are the zeros of the Hermite polynomial $H_{k}(\xi)$. Clearly, at an initial time the position of the zeros and poles is $q_{k}^{(l)}(0)=\tau_{k}^{(l)} \sqrt{\hbar / \omega_{0}}$, and when $\gamma>0, t \rightarrow \infty$ one has $q_{k}^{(l)}(t) \rightarrow 0$ due to increasing mass $\mu(t)=e^{\gamma t}$ (dissipation). 


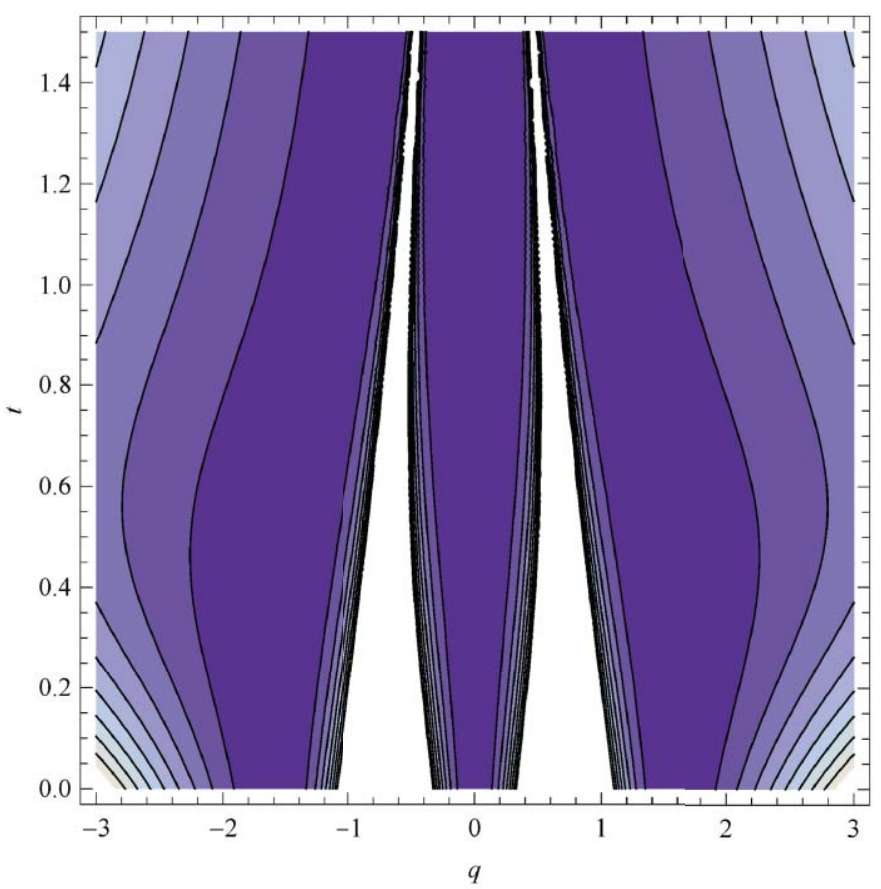

(a)

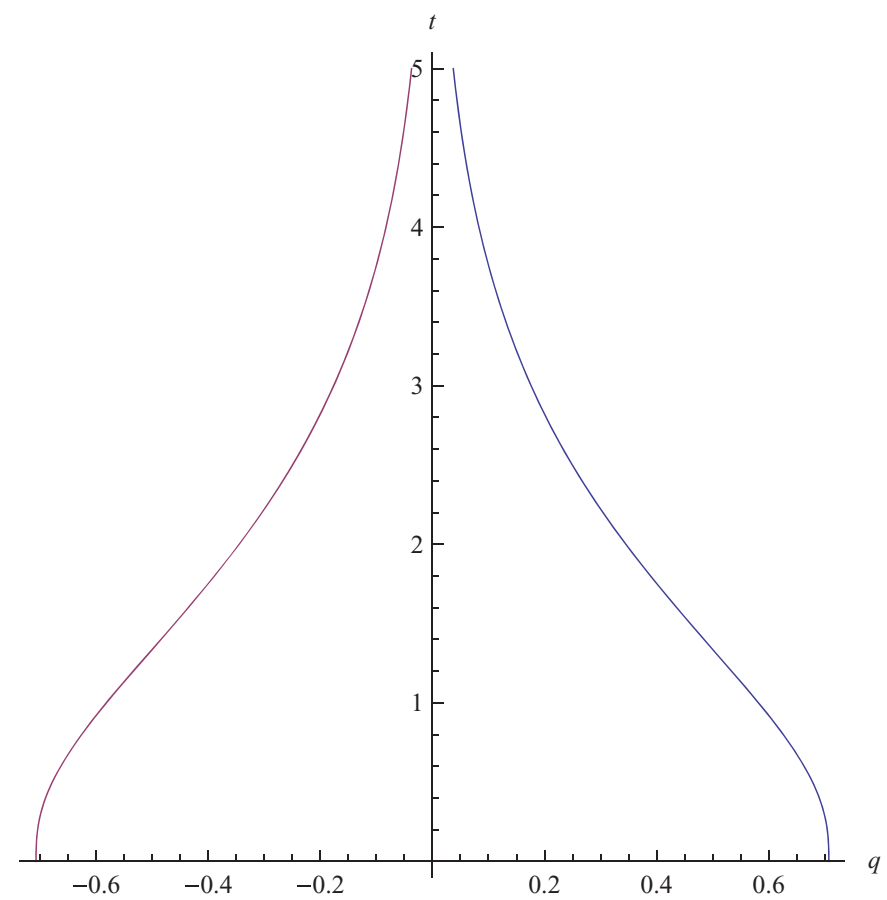

(b)

FIG. 5. Case $\Omega^{2}=0$. (a) Contour plot of $\left|V_{2}(q, t)\right|^{2}$. (b) Position of the moving poles of $\left|V_{2}(q, t)\right|^{2}$ ( zeros of $\rho_{2}(q, t)$,) described by the curves $q_{2}^{(1)}(t)=-\frac{e^{-t}}{\sqrt{2}} \sqrt{(1+t)^{2}+t^{2}}$ and $q_{2}^{(2)}(t)=-q_{2}^{(1)}(t)$.

In Figs. 1, 2, and 3, we illustrate the behavior of $\rho_{k}(q, t)$ for $k=0,1,2$ and in Fig. 4 we plot $\left|V_{2}(q, t)\right|^{2}$. Figure 5 shows the motion of zeros and poles for $k=2$. For simplicity, the constants are chosen to be $x_{0}=\hbar=\omega_{0}=1$ and $\gamma=2$. 


\section{Under damping case, $\Omega^{2}>0$}

If $\Omega^{2}=\omega_{0}^{2}-\left(\gamma^{2} / 4\right)>0$, then the classical equation (42) has solution

$$
x_{2}(t)=x_{0} \frac{\omega_{0}}{|\Omega|} e^{-\frac{\gamma t}{2}} \cos [|\Omega| t-\alpha],
$$

where $\alpha=\tan ^{-1}\left(\frac{\gamma}{2|\Omega|}\right)$, and the linear Schrödinger equation in (41) has exact solutions of the form

$$
\begin{aligned}
\Psi_{k}(q, t)= & N_{k} \sqrt{R_{2}(t)} \times \exp \left(i\left(k+\frac{1}{2}\right) \arctan \left(\frac{\omega_{0}}{\hbar} g_{2}(t)\right)\right) \\
& \times \exp \left(i\left(\frac{e^{\gamma t} \dot{x}_{2}(t)}{2 \hbar x_{2}(t)}-\frac{1}{2}\left(\frac{\omega_{0}}{\hbar}\right)^{2} g_{2}(t) R_{2}^{2}(t)\right) q^{2}\right) \\
& \times \exp \left(-\frac{1}{2} \frac{\omega_{0}}{\hbar} R_{2}^{2}(t) q^{2}\right) \times H_{k}\left(\sqrt{\frac{\omega_{0}}{\hbar}} R_{2}(t) q\right),
\end{aligned}
$$

where

$$
\begin{gathered}
g_{2}(t)=-\hbar \frac{|\Omega|}{\omega_{0}^{2}}\left(\tan [|\Omega| t-\alpha]+\frac{\gamma}{2|\Omega|}\right) \\
R_{2}(t)=\frac{|\Omega|}{\omega_{0}} \frac{e^{\frac{\gamma t}{2}}}{|\cos [|\Omega| t-\alpha]|}\left(1+\frac{\Omega^{2}}{\omega_{0}^{2}}\left(\tan [|\Omega| t-\alpha]+\frac{\gamma}{2|\Omega|}\right)^{2}\right)^{-1 / 2} .
\end{gathered}
$$

a. Hamilton-Jacobi-type representation, $\Omega^{2}>0$. The nonlinear system consisting of generalized Hamilton-Jacobi equation and continuity equation

$$
\left\{\begin{array}{l}
\frac{\partial S}{\partial t}+\frac{1}{2} e^{-\gamma t}\left(\frac{\partial S}{\partial q}\right)^{2}+\frac{\omega_{0}^{2}}{2} e^{\gamma t} q^{2}=\frac{\hbar^{2}}{2} e^{-\gamma t}\left[\frac{1}{\sqrt{\rho}} \frac{\partial^{2} \sqrt{\rho}}{\partial q^{2}}\right], \\
\frac{\partial \rho}{\partial t}+e^{-\gamma t} \frac{\partial}{\partial q}\left[\rho \frac{\partial S}{\partial q}\right]=0
\end{array}\right.
$$

with specific initial conditions

$$
S_{k}(q, 0)=0, \quad \rho_{k}(q, 0)=N_{k}^{2} \exp \left(-\frac{\omega_{0}}{\hbar} q^{2}\right) H_{k}^{2}\left(\sqrt{\frac{\omega_{0}}{\hbar}} q\right), \quad k=0,1,2, \ldots,
$$

has exact solutions of the form

$$
\begin{gathered}
S_{k}(q, t)=\frac{1}{2}\left(e^{\gamma t} \frac{\dot{x}_{2}(t)}{x_{2}(t)}-\frac{\omega_{0}^{2}}{\hbar} g_{2}(t) R_{2}^{2}(t)\right) q^{2}+\hbar\left(k+\frac{1}{2}\right) \arctan \left(\frac{\omega_{0}}{\hbar} g_{2}(t)\right), \\
\rho_{k}(q, t)=N_{k}^{2} \times R_{2}(t) \times \exp \left(-\frac{\omega_{0}}{\hbar} R_{2}^{2}(t) q^{2}\right) \times H_{k}^{2}\left(\sqrt{\frac{\omega_{0}}{\hbar}} R_{2}(t) q\right) .
\end{gathered}
$$

b. Madelung hydrodynamic equations, $\Omega^{2}>0$. The system of hydrodynamic equations

$$
\left\{\begin{array}{l}
\frac{\partial v}{\partial t}+\gamma v+v \frac{\partial v}{\partial q}=-e^{-\gamma t} \frac{\partial}{\partial q}\left[\frac{-\hbar^{2}}{2} \frac{e^{-\gamma t}}{\sqrt{\rho}} \frac{\partial^{2} \sqrt{\rho}}{\partial q^{2}}+\frac{\omega_{0}^{2}}{2} e^{\gamma t} q^{2}\right], \\
\frac{\partial \rho}{\partial t}+\frac{\partial}{\partial q}[\rho v]=0,
\end{array}\right.
$$

with specific initial conditions

$$
v_{k}(q, 0)=0, \quad \rho_{k}(q, 0)=N_{k}^{2} \exp \left(-\frac{\omega_{0}}{\hbar} q^{2}\right) H_{k}^{2}\left(\sqrt{\frac{\omega_{0}}{\hbar}} q\right), \quad k=0,1,2, \ldots
$$

has solutions

$$
v_{k}(q, t)=\left(\frac{\dot{x}_{2}(t)}{x_{2}(t)}-\frac{\omega_{0}^{2}}{\hbar} e^{-\gamma t} g_{2}(t) R_{2}^{2}(t)\right) q
$$

and $\rho_{k}(q, t)$ given by (54). 


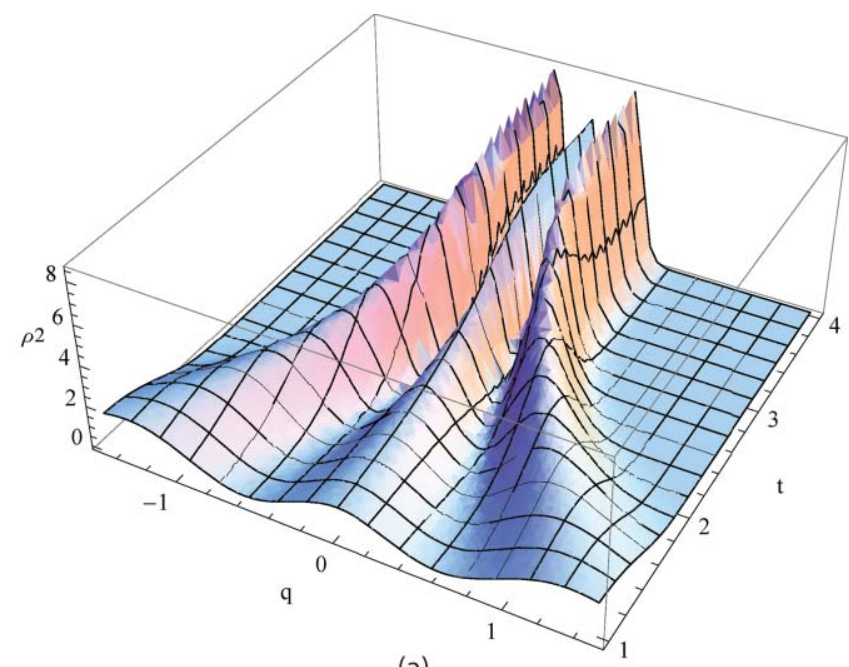

(a)

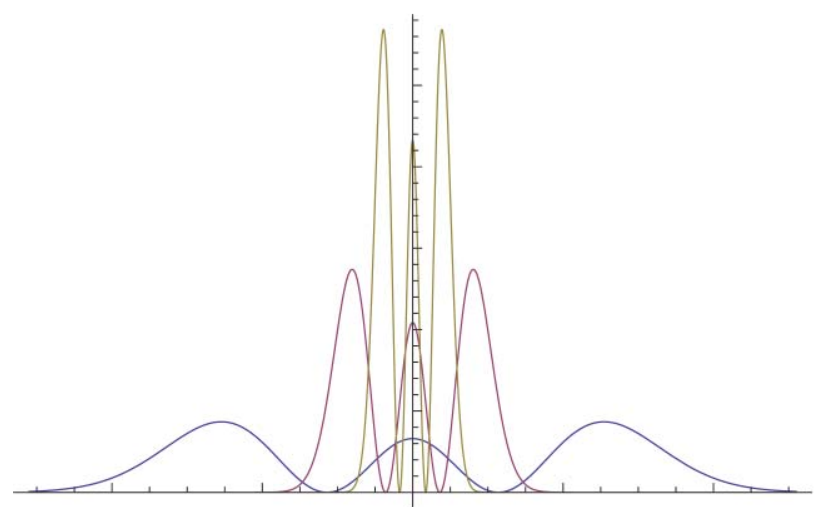

(b)

FIG. 6. Case $\Omega^{2}>0$. (a) Evolution of probability density $\rho_{2}(q, t)$. (b) Behavior of $\rho_{2}(q, t)$ at times $t=4,6,8$.

c. The potential Schrödinger-Burgers equation, $\Omega^{2}>0$. The IVP for the potential SchrödingerBurgers equation

$$
\left\{\begin{array}{l}
\frac{\partial F}{\partial t}+\gamma F+\frac{1}{2}\left(\frac{\partial F}{\partial q}\right)^{2}+\frac{\omega_{0}^{2}}{2} q^{2}=i \hbar \frac{e^{-\gamma t}}{2}\left(\frac{\partial^{2} F}{\partial q^{2}}\right), \\
F_{k}(q, 0)=i\left(\frac{\omega_{0}}{2} q^{2}-\hbar \ln \left(N_{k} H_{k}\left(\sqrt{\frac{\omega_{0}}{\hbar}} q\right)\right)\right), \quad k=0,1,2, \ldots
\end{array}\right.
$$

has exact solutions of the form

$$
F_{k}(q, t)=\mathrm{F}_{1, k}(q, t)+i \mathrm{~F}_{2, k}(q, t),
$$

where

$$
\begin{gathered}
\mathrm{F}_{1, k}(q, t)=\left[\frac{1}{2}\left(\frac{\dot{x}_{2}(t)}{x_{2}(t)}-\frac{\omega_{0}^{2}}{\hbar} e^{-\gamma t} g_{2}(t) R_{2}^{2}(t)\right) q^{2}+\hbar e^{-\gamma t}\left(k+\frac{1}{2}\right) \arctan \left(\frac{\omega_{0}}{\hbar} g_{2}(t)\right)\right] \\
\mathrm{F}_{2, k}(q, t)=e^{-\gamma t}\left[\frac{\omega_{0}}{2} R_{2}^{2}(t) q^{2}-\hbar \ln \left(N_{k} \sqrt{R_{2}(t)} H_{k}\left(\sqrt{\frac{\omega_{0}}{\hbar}} R_{2}(t) q\right)\right)\right] .
\end{gathered}
$$




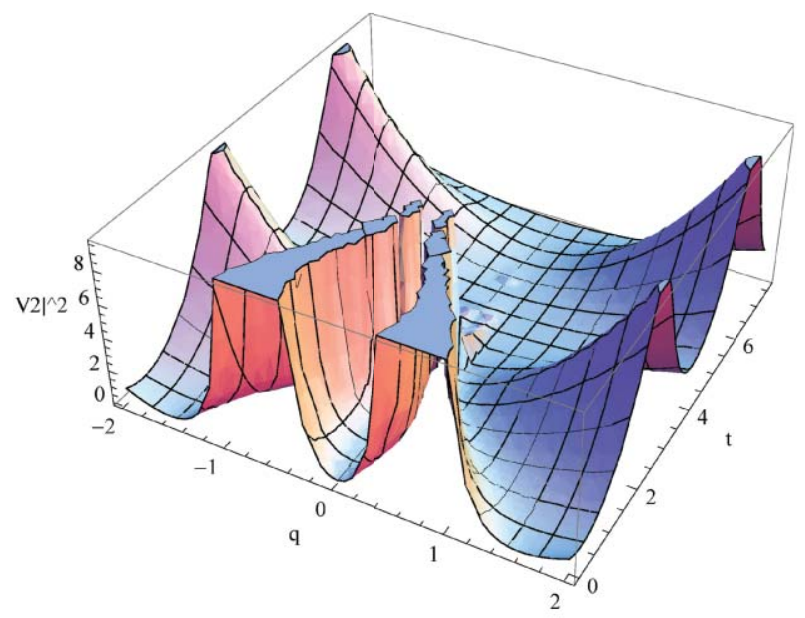

(a)

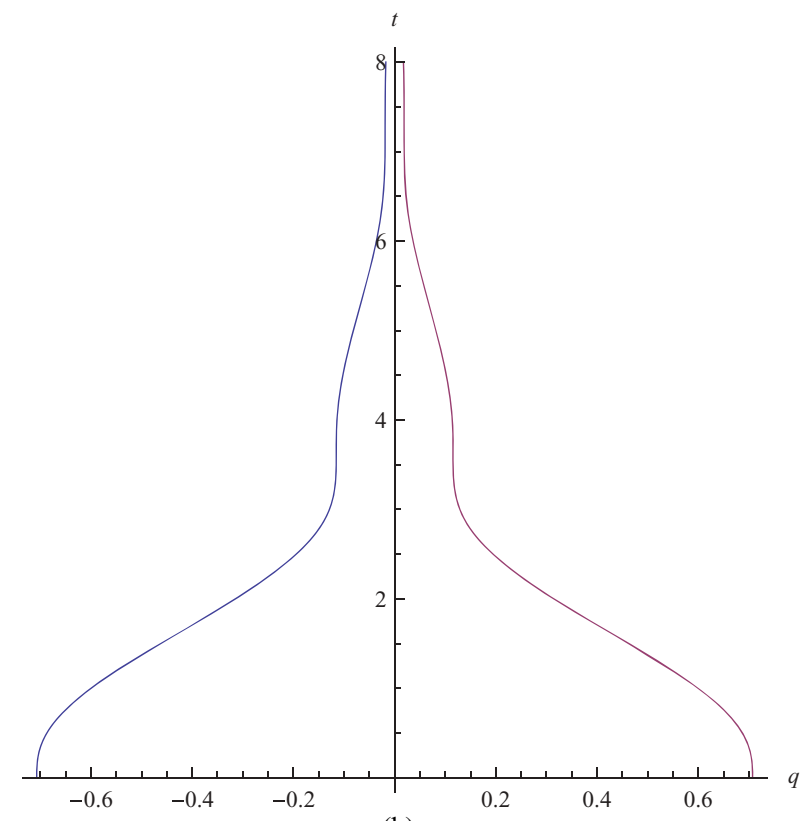

(b)

FIG. 7. Case $\Omega^{2}>0$. (a) Evolution of $\left|V_{2}(q, t)\right|^{2}$. (b) Position of the moving poles of $\left|V_{2}(q, t)\right|^{2}$, described by $q_{2}^{(1)}(t)=$ $-\sqrt{\frac{2}{3}} \cos \left[\frac{\sqrt{3}}{2} t-\frac{\pi}{6}\right] \times e^{-\frac{t}{2}} \sqrt{1+\frac{3}{4}\left(\tan \left[\frac{\sqrt{3}}{2} t-\frac{\pi}{6}\right]+\frac{1}{\sqrt{3}}\right)^{2}}, \quad q_{2}^{(2)}(t)=-q_{2}^{(1)}(t)$.

The real and imaginary parts of $F_{k}$, that is $\mathrm{F}_{1, k}$ and $\mathrm{F}_{2, k}$ are solutions of the IVP

$$
\left\{\begin{array}{l}
\frac{\partial \mathrm{F}_{1}}{\partial t}+\gamma \mathrm{F}_{1}+\frac{1}{2}\left(\left(\frac{\partial \mathrm{F}_{1}}{\partial q}\right)^{2}-\left(\frac{\partial \mathrm{F}_{2}}{\partial q}\right)^{2}\right)+\frac{\omega_{0}^{2}}{2} q^{2}=-\hbar \frac{e^{-\gamma t}}{2} \frac{\partial^{2} \mathrm{~F}_{2}}{\partial q^{2}} \\
\frac{\partial \mathrm{F}_{2}}{\partial t}+\gamma \mathrm{F}_{2}+\frac{\partial \mathrm{F}_{1}}{\partial q} \frac{\partial \mathrm{F}_{2}}{\partial q}=\hbar \frac{e^{-\gamma t}}{2} \frac{\partial^{2} \mathrm{~F}_{1}}{\partial q^{2}} \\
\mathrm{~F}_{1}(q, 0)=0, \quad \mathrm{~F}_{2}(q, 0)=\left(\frac{\omega_{0}}{2} q^{2}-\hbar \ln \left(N_{k} H_{k}\left(\sqrt{\frac{\omega_{0}}{\hbar}} q\right)\right)\right) .
\end{array}\right.
$$


d. The Schrödinger-Burgers equation, $\Omega^{2}>0$. The IVP for the Schrödinger-Burgers equation

$$
\left\{\begin{array}{l}
\frac{\partial V}{\partial t}+\gamma V+V \frac{\partial V}{\partial q}+\omega_{0}^{2} q=i \hbar \frac{e^{-\gamma t}}{2} \frac{\partial^{2} V}{\partial q^{2}} \\
V_{k}(q, 0)=i\left[\omega_{0} q-2 k \sqrt{\hbar \omega_{0}}\left(\frac{H_{k-1}\left(\sqrt{\frac{\omega_{0}}{\hbar}} q\right)}{H_{k}\left(\sqrt{\frac{\omega_{0}}{\hbar}} q\right)}\right)\right], k=0,1,2, \ldots,
\end{array}\right.
$$

has solutions

$$
\begin{aligned}
V_{k}(q, t)= & {\left[\frac{\dot{x}_{2}(t)}{x_{2}(t)}-\frac{\omega_{0}^{2}}{\hbar} e^{-\gamma t} g_{2}(t) R_{2}^{2}(t)\right] q } \\
& +i e^{-\gamma t}\left[\omega_{0} R_{2}^{2}(t) q-2 k \sqrt{\omega_{0} \hbar} R_{2}(t)\left(\frac{H_{k-1}\left(\sqrt{\frac{\omega_{0}}{\hbar}} R_{2}(t) q\right)}{H_{k}\left(\sqrt{\frac{\omega_{0}}{\hbar}} R_{2}(t) q\right)}\right)\right] .
\end{aligned}
$$

Therefore, the system for the classical and quantum velocities

$$
\left\{\begin{array}{l}
\frac{\partial v}{\partial t}+\gamma v+v \frac{\partial v}{\partial q}+\omega_{0}^{2} q=-\frac{\hbar}{2} e^{-\gamma t} \frac{\partial^{2} u}{\partial q^{2}}+u \frac{\partial u}{\partial q} \\
\frac{\partial u}{\partial t}+\gamma u+u \frac{\partial v}{\partial q}+u \frac{\partial v}{\partial q}=\frac{\hbar}{2} e^{-\gamma t} \frac{\partial^{2} v}{\partial q^{2}}
\end{array}\right.
$$

with initial conditions

$$
v_{k}(q, 0)=0, \quad u_{k}(q, 0)=\omega_{0} q-2 k \sqrt{\hbar \omega_{0}}\left(\frac{H_{k-1}\left(\sqrt{\frac{\omega_{0}}{\hbar}} q\right)}{H_{k}\left(\sqrt{\frac{\omega_{0}}{\hbar}} q\right)}\right), \quad k=0,1,2, \ldots,
$$

has solutions $v_{k}(q, t)$ and $u_{k}(q, t)$ which are respectively, the real and imaginary parts of $V_{k}(q, t)$ given by expression (55).

e. Motion of zeros and poles, $\Omega^{2}>0$. From expression (51), we see that $\Psi_{k}(q, t)$ has zeros at points where $H_{k}\left(\sqrt{\Omega_{0}} R_{2}(t) q\right)=0$ and these zeros are poles of the solution $V_{k}(q, t)$ for the nonlinear Schrödinger-Burgers equation. Then, we find that for each $k=1,2,3, \ldots$, the motion of the zeros and poles is described explicitly by

$q_{k}^{(l)}(t)=\tau_{k}^{(l)} \frac{\sqrt{\hbar \omega_{0}}}{|\Omega|} \times e^{-\frac{\gamma}{2} t} \times \mid \cos [|\Omega| t-\alpha] \sqrt{1+\frac{\Omega^{2}}{\omega_{0}^{2}}\left(\tan [|\Omega| t-\alpha]+\frac{\gamma}{2|\Omega|}\right)^{2}}, \quad l=1,2, \ldots k$.

As before, for $\gamma>0$ and $t \rightarrow \infty$ we have $q_{k}^{(l)}(t) \rightarrow 0$ due to dissipation.

As an example for the underdamping case, in Fig. 6 we give the plot of $\rho_{2}(q, t)$, and in Fig. 7 we illustrate $\left|V_{2}(q, t)\right|^{2}$, and the behavior of its poles. The constants are chosen to be $x_{0}=\hbar=\omega_{0}$ $=\gamma=1$.

\section{Over damping case, $\Omega^{2}<0$}

When $\Omega^{2}=\omega_{0}^{2}-\left(\gamma^{2} / 4\right)<0$, the classical equation (42) has solution:

$$
x_{3}(t)=x_{0} \frac{\omega_{0}}{\Omega^{\prime}} e^{-\frac{\gamma t}{2}} \sinh \left[\Omega^{\prime} t+\beta\right]
$$




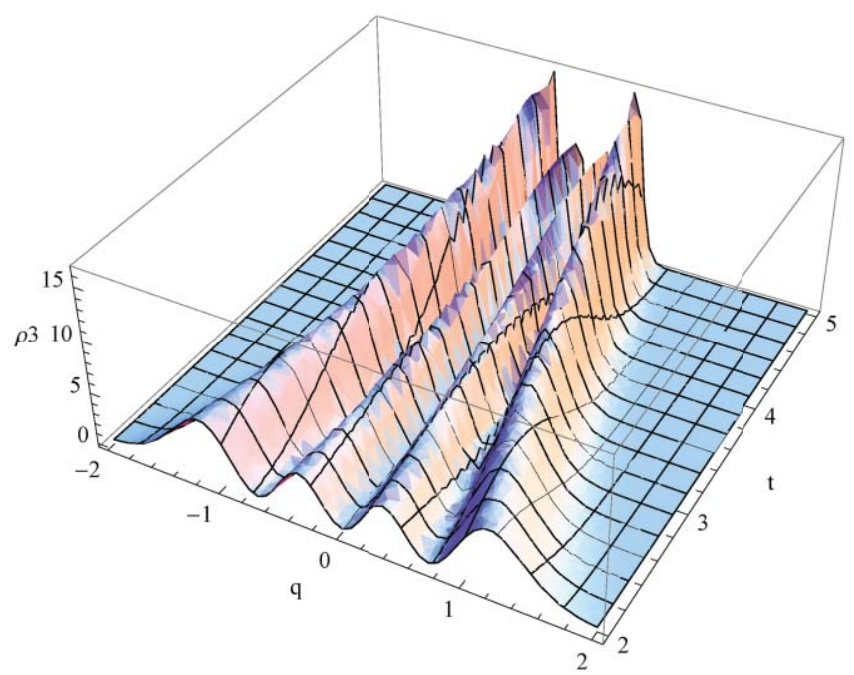

(a)

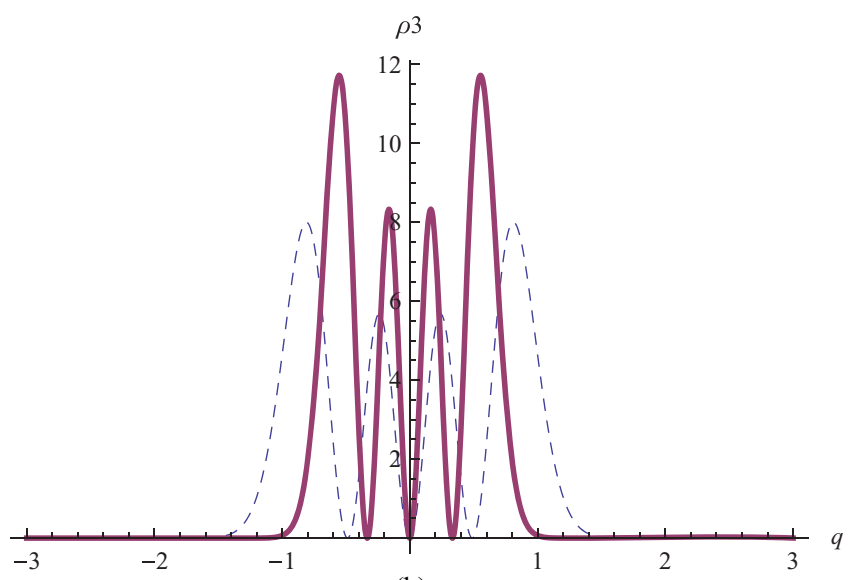

(b)

FIG. 8. Case $\Omega^{2}<0$. (a) Evolution of probability density $\rho_{3}(q, t)$. (b) Behavior of $\rho_{3}(q, t)$ at times $t=3,4$.

where $\Omega^{\prime}=\sqrt{\left|\Omega^{2}\right|}$, and $\beta=\operatorname{coth}^{-1}\left(\frac{\gamma}{2 \Omega^{\prime}}\right)$. Then, the linear Schrödinger equation in (41) has exact solutions:

$$
\begin{aligned}
& \Psi_{k}(q, t)=N_{k} \sqrt{R_{3}(t)} \times \exp \left(i\left(k+\frac{1}{2}\right) \arctan \left(\frac{\omega_{0}}{\hbar} g_{3}(t)\right)\right) \\
& \quad \times \exp \left(i\left(\frac{e^{\gamma t} \dot{x}_{3}(t)}{2 \hbar x_{3}(t)}-\frac{1}{2}\left(\frac{\omega_{0}}{\hbar}\right)^{2} g_{3}(t) R_{3}^{2}(t)\right) q^{2}\right) \times \exp \left(-\frac{1}{2} \frac{\omega_{0}}{\hbar} R_{3}^{2}(t) q^{2}\right) \times H_{k}\left(\sqrt{\frac{\omega_{0}}{\hbar}} R_{3}(t) q\right),
\end{aligned}
$$

where

$$
\begin{gathered}
g_{3}(t)=\frac{\hbar \Omega^{\prime}}{\omega_{0}^{2}}\left(\operatorname{coth}\left[\Omega^{\prime} t+\beta\right]-\frac{\gamma}{2 \Omega^{\prime}}\right) \\
R_{3}(t)=\frac{\Omega^{\prime}}{\omega_{0}} \frac{e^{\frac{\gamma t}{2}}}{\left|\sinh \left[\Omega^{\prime} t+\beta\right]\right|}\left(1+\frac{\Omega^{\prime 2}}{\omega_{0}^{2}}\left(\operatorname{coth}\left[\Omega^{\prime} t+\beta\right]-\frac{\gamma}{2 \Omega^{\prime}}\right)^{2}\right)^{-1 / 2} .
\end{gathered}
$$




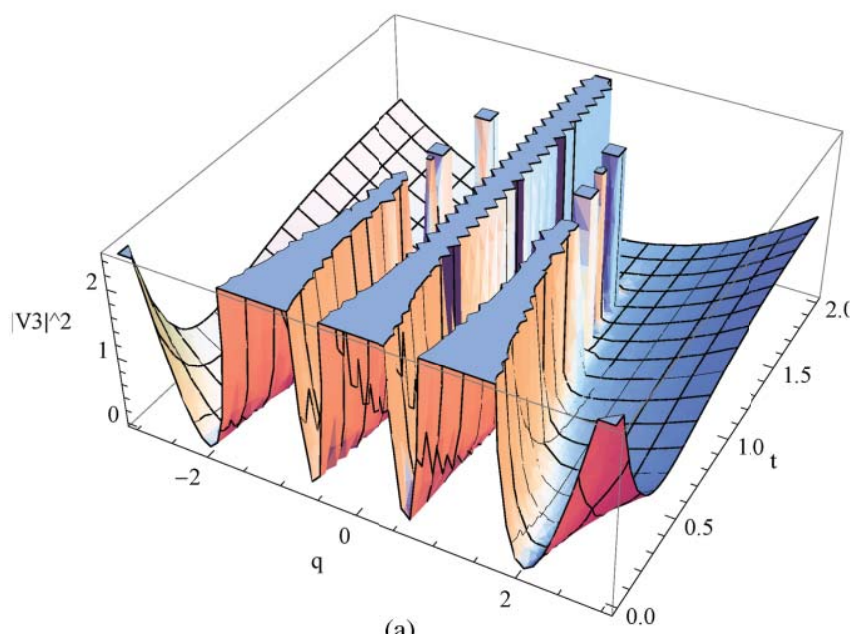

(a)

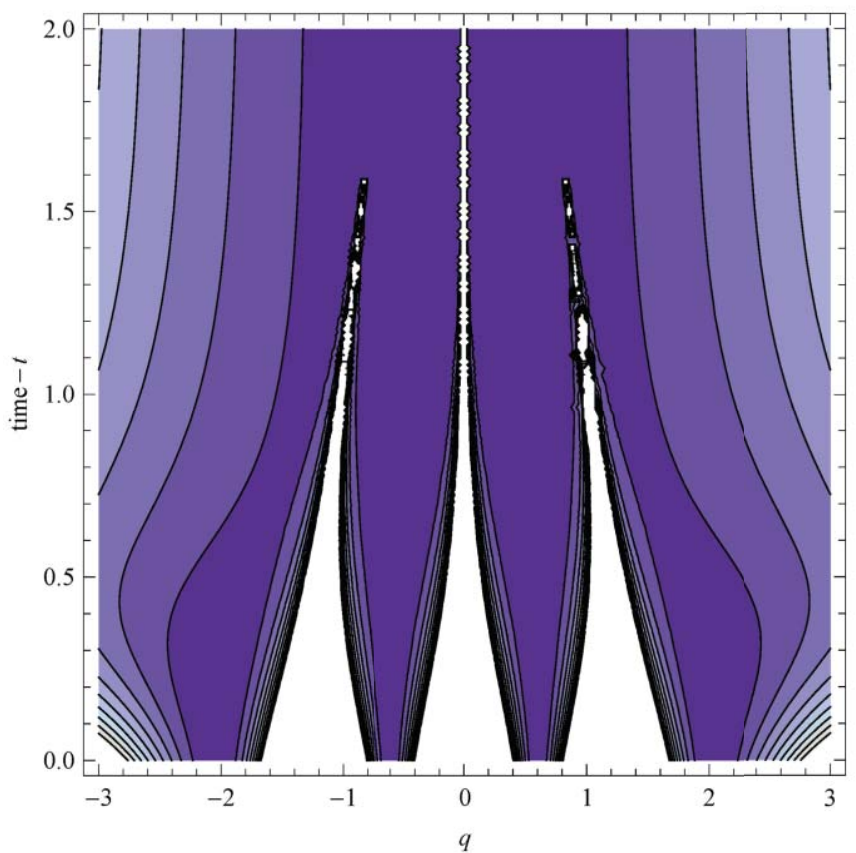

(b)

FIG. 9. Case $\Omega^{2}<0$. (a) Evolution of $\left|V_{3}(q, t)\right|^{2}$. (b) Contour plot of $\left|V_{3}(q, t)\right|^{2}$, from which one can observe the position $q_{3}^{(1)}(t), q_{3}^{(2)}(t)$, and $q_{3}^{(3)}(t)$ of the moving poles.

a. Hamilton-Jacobi-type representation of Caldirola-Kanai oscillator, $\Omega^{2}<0$. The nonlinear system consisting of generalized Hamilton-Jacobi equation and continuity equation:

$$
\left\{\begin{array}{l}
\frac{\partial S}{\partial t}+\frac{1}{2} e^{-\gamma t}\left(\frac{\partial S}{\partial q}\right)^{2}+\frac{\omega_{0}^{2}}{2} e^{\gamma t} q^{2}=\frac{\hbar^{2}}{2} e^{-\gamma t}\left[\frac{1}{\sqrt{\rho}} \frac{\partial^{2} \sqrt{\rho}}{\partial q^{2}}\right], \\
\frac{\partial \rho}{\partial t}+e^{-\gamma t} \frac{\partial}{\partial q}\left[\rho \frac{\partial S}{\partial q}\right]=0,
\end{array}\right.
$$

with specific initial conditions

$$
S(q, 0)=0, \quad \rho_{k}(q, 0)=N_{k}^{2} \exp \left(-\frac{\omega_{0}}{\hbar} q^{2}\right) H_{k}^{2}\left(\sqrt{\frac{\omega_{0}}{\hbar}} q\right), \quad k=0,1,2, \ldots,
$$


has exact solutions of the form

$$
\begin{gathered}
S_{k}(q, t)=\frac{1}{2}\left(e^{\gamma t} \frac{\dot{x}_{3}(t)}{x_{3}(t)}-\frac{\omega_{0}^{2}}{\hbar} g_{3}(t) R_{3}^{2}(t)\right) q^{2}+\hbar\left(k+\frac{1}{2}\right) \arctan \left(\frac{\omega_{0}}{\hbar} g_{3}(t)\right), \\
\rho_{k}(q, t)=N_{k}^{2} \times R_{3}(t) \times \exp \left(-\frac{\omega_{0}}{\hbar} R_{3}^{2}(t) q^{2}\right) \times H_{k}^{2}\left(\sqrt{\frac{\omega_{0}}{\hbar}} R_{3}(t) q\right) .
\end{gathered}
$$

b. Madelung hydrodynamic equations, $\Omega^{2}<0$. The system of hydrodynamic equations

$$
\left\{\begin{aligned}
\frac{\partial v}{\partial t}+\gamma v+v \frac{\partial v}{\partial q} & =-e^{-\gamma t} \frac{\partial}{\partial q}\left[\frac{-\hbar^{2}}{2} \frac{e^{-\gamma t}}{\sqrt{\rho}} \frac{\partial^{2} \sqrt{\rho}}{\partial q^{2}}+\frac{\omega_{0}^{2}}{2} e^{\gamma t} q^{2}\right], \\
\frac{\partial \rho}{\partial t}+\frac{\partial}{\partial q}[\rho v] & =0,
\end{aligned}\right.
$$

with specific initial conditions

$$
v_{k}(q, 0)=0, \quad \rho_{k}(q, 0)=N_{k}^{2} \exp \left(-\frac{\omega_{0}}{\hbar} q^{2}\right) H_{k}^{2}\left(\sqrt{\frac{\omega_{0}}{\hbar}} q\right), \quad k=0,1,2, \ldots
$$

has solutions

$$
v_{k}(q, t)=\left(\frac{\dot{x}_{3}(t)}{x_{3}(t)}-\frac{\omega_{0}^{2}}{\hbar} e^{-\gamma t} g_{3}(t) R_{3}^{2}(t)\right) q
$$

and $\rho_{k}(q, t)$ given by (59).

c. The potential Schrödinger-Burgers equation, $\Omega^{2}<0$. The IVP for potential SchrödingerBurgers equation

$$
\left\{\begin{array}{l}
\frac{\partial F}{\partial t}+\gamma F+\frac{1}{2}\left(\frac{\partial F}{\partial q}\right)^{2}+\frac{\omega_{0}^{2}}{2} q^{2}=i \hbar \frac{e^{-\gamma t}}{2}\left(\frac{\partial^{2} F}{\partial q^{2}}\right), \\
F_{k}(q, 0)=i\left(\frac{\omega_{0}}{2} q^{2}-\hbar \ln \left(N_{k} H_{k}\left(\sqrt{\frac{\omega_{0}}{\hbar}} q\right)\right)\right), \quad k=0,1,2, \ldots
\end{array}\right.
$$

has exact solutions of the form

$$
F_{k}(q, t)=\mathrm{F}_{1, k}(q, t)+i \mathrm{~F}_{2, k}(q, t),
$$

where

$$
\begin{gathered}
\mathrm{F}_{1, k}(q, t)=\left[\frac{1}{2}\left(\frac{\dot{x}_{3}(t)}{x_{3}(t)}-\frac{\omega_{0}^{2}}{\hbar} e^{-\gamma t} g_{3}(t) R_{3}^{2}(t)\right) q^{2}+\hbar e^{-\gamma t}\left(k+\frac{1}{2}\right) \arctan \left(\frac{\omega_{0}}{\hbar} g_{3}(t)\right)\right] \\
\mathrm{F}_{2, k}(q, t)=e^{-\gamma t}\left[\frac{\omega_{0}}{2} R_{3}^{2}(t) q^{2}-\hbar \ln \left(N_{k} \sqrt{R_{3}(t)} H_{k}\left(\sqrt{\frac{\omega_{0}}{\hbar}} R_{3}(t) q\right)\right)\right] .
\end{gathered}
$$

The real and imaginary parts of $F_{k}$, that is $\mathrm{F}_{1, k}$ and $\mathrm{F}_{2, k}$, are solutions of the IVP :

$$
\left\{\begin{array}{l}
\frac{\partial \mathrm{F}_{1}}{\partial t}+\gamma \mathrm{F}_{1}+\frac{1}{2}\left(\left(\frac{\partial \mathrm{F}_{1}}{\partial q}\right)^{2}-\left(\frac{\partial \mathrm{F}_{2}}{\partial q}\right)^{2}\right)+\frac{\omega_{0}^{2}}{2} q^{2}=-\hbar \frac{e^{-\gamma t}}{2} \frac{\partial^{2} \mathrm{~F}_{2}}{\partial q^{2}} \\
\frac{\partial \mathrm{F}_{2}}{\partial t}+\gamma \mathrm{F}_{2}+\frac{\partial \mathrm{F}_{1}}{\partial q} \frac{\partial \mathrm{F}_{2}}{\partial q}=\hbar \frac{e^{-\gamma t}}{2} \frac{\partial^{2} \mathrm{~F}_{1}}{\partial q^{2}} \\
\mathrm{~F}_{1, k}(q, 0)=0, \quad \mathrm{~F}_{2, k}(q, 0)=\left(\frac{\omega_{0}}{2} q^{2}-\hbar \ln \left(N_{k} H_{k}\left(\sqrt{\frac{\omega_{0}}{\hbar}} q\right)\right)\right) .
\end{array}\right.
$$


d. The Schrödinger-Burgers equation, $\Omega^{2}<0$. The IVP for the nonlinear Schrödinger-Burgers equation

$$
\left\{\begin{array}{l}
\frac{\partial V}{\partial t}+\gamma V+V \frac{\partial V}{\partial q}+\omega_{0}^{2} q=i \hbar \frac{e^{-\gamma t}}{2} \frac{\partial^{2} V}{\partial q^{2}} \\
V_{k}(q, 0)=i\left[\omega_{0} q-2 k \sqrt{\omega_{0} \hbar}\left(\frac{H_{k-1}\left(\sqrt{\frac{\omega_{0}}{\hbar}} q\right)}{\left.H_{k}\left(\sqrt{\left.\frac{\omega_{0}}{\hbar} q\right)}\right)\right], \quad k=0,1,2, \ldots,}\right.\right.
\end{array}\right.
$$

has solutions

$$
\begin{aligned}
V_{k}(q, t)= & {\left[\frac{\dot{x}_{3}(t)}{x_{3}(t)}-\frac{\omega_{0}^{2}}{\hbar} R_{3}(t) e^{-\gamma t} g_{3}(t) R_{3}^{2}(t)\right] q+i e^{-\gamma t} } \\
& \times\left[\omega_{0} R_{3}^{2}(t) q-2 k \sqrt{\omega_{0} \hbar} R_{3}(t)\left(\frac{H_{k-1}\left(\sqrt{\frac{\omega_{0}}{\hbar}} R_{3}(t) q\right)}{H_{k}\left(\sqrt{\frac{\omega_{0}}{\hbar}} R_{3}(t) q\right)}\right)\right]
\end{aligned}
$$

Obviously, the system for the classical and quantum velocities

$$
\left\{\begin{array}{l}
\frac{\partial v}{\partial t}+\gamma v+v \frac{\partial v}{\partial q}+\omega_{0}^{2} q=-\hbar \frac{e^{-\gamma t}}{2} \frac{\partial^{2} u}{\partial q^{2}}+u \frac{\partial u}{\partial q} \\
\frac{\partial u}{\partial t}+\gamma u+u \frac{\partial v}{\partial q}+u \frac{\partial v}{\partial q}=\hbar \frac{e^{-\gamma t}}{2} \frac{\partial^{2} v}{\partial q^{2}}
\end{array}\right.
$$

with initial conditions

$$
v_{k}(q, 0)=0, \quad u_{k}(q, 0)=\left[\omega_{0} q-\sqrt{\omega_{0} \hbar}\left(\frac{H_{k-1}\left(\frac{\omega_{0}}{\hbar} q\right)}{H_{k}\left(\sqrt{\frac{\omega_{0}}{\hbar}} q\right)}\right)\right], \quad k=0,1,2, \ldots,
$$

has solutions $v(q, t)$ and $u_{k}(q, t)$ which are the real and imaginary parts of $V_{k}(q, t)$ given by (64).

$e$. Motion of zeros and poles, $\Omega^{2}<0$. The wave function $\Psi_{k}(q, t)$ given by $(58)$ has zeros at points where $H_{k}\left(\sqrt{\frac{\omega_{0}}{\hbar}} R_{3}(t) q\right)=0$ and these zeros are poles of the solution $V_{k}(q, t)$ for the nonlinear Schrödinger-Burgers equation (63). For each $k=1,2,3, \ldots$, the motion of the zeros and poles is described explicitly by

$q_{k}^{(l)}(t)=\tau_{k}^{(l)} \sqrt{\frac{\hbar}{\omega_{0}}} \times e^{-\frac{\gamma}{2} t} \times\left|\sinh \left[\Omega^{\prime} t+\beta\right]\right| \sqrt{1+\frac{\Omega^{\prime 2}}{\omega_{0}^{2}}\left(\operatorname{coth}\left[\Omega^{\prime} t+\beta\right]-\frac{\gamma}{2 \Omega^{\prime}}\right)^{2}}, \quad l=1,2, \ldots k$.

As an example, we illustrate the evolution of the probability density $\rho_{3}(q, t)$ in Fig. 8 , and $\left|V_{3}(q, t)\right|^{2}$ in Fig. $9\left(x_{0}=\hbar=\omega_{0}=1, \gamma=3\right)$. In that case, according to $(66),\left|V_{3}(q, t)\right|^{2}$ has three moving poles, whose position is described by $q_{3}^{(1)}(t), q_{3}^{(2)}(t)=0$, and $q_{3}^{(3)}(t)=-q_{3}^{(1)}(t)$, where

$$
q_{3}^{(1)}(t)=-\sqrt{\frac{2}{3}} \times e^{-\frac{3}{2} t} \times \cosh \left[\frac{\sqrt{5}}{2} t\right] \sqrt{\left(1+\frac{3}{\sqrt{5}} \tanh \left[\frac{\sqrt{5}}{2} t\right]\right)^{2}+\left(\frac{2}{\sqrt{5}} \tanh \left[\frac{\sqrt{5}}{2} t\right]\right)^{2}} .
$$

\section{DIRAC-DELTA BEHAVIOR OF PROBABILITY DENSITY AT TIME INFINITY}

In Sec. V, explicit formulas for probability density functions $\rho_{k}(q, t)$ of the Caldirola-Kanai oscillator were given, and some illustrative plots were constructed. We observe that the functions $\rho_{k}(q, t)$ at time infinity, behave like Dirac-delta distribution $\delta(q)$. Moreover, from Figs. 1, 3, and 6, we see that when $k$ is even, at $q=0$ one has $\rho_{k}(0, t) \rightarrow \infty$ as $t \rightarrow \infty$, which is a typical behavior of the well known delta sequences. However, when $k$ is odd, at $q=0$ one has $\rho_{k}(0, t)=0$ for all $t$, see Figs. 2 and 8. This is an unusual property and recently it was discussed also in (Ref. 16). In 
what follows, we state the result more precisely. Consider the probability density function (11) for the Schrödinger equation (1), that is

$\rho_{k}(q, t)=\frac{1}{2^{k} k ! \sqrt{\pi}} \times \sqrt{\Omega_{0}} R(t) \times \exp \left(-\left(\sqrt{\Omega_{0}} R(t) q\right)^{2}\right) \times H_{k}^{2}\left(\sqrt{\Omega_{0}} R(t) q\right), \quad k=0,1,2, \ldots$,

and in particular assume that

$$
\lim _{t \rightarrow \infty} R(t)=\infty
$$

which can be easily verified for the Caldirola-Kanai oscillator. Then, we claim that, for each fixed $k=0,1,2, \ldots$, the probability densities $\rho_{k}(q, t)$, considered as functions of a single variable $q$, with parameter $t>0$, form a delta-convergent sequence, that is

$$
\lim _{t \rightarrow \infty} \rho_{k}(q, t)=\delta(q)
$$

Indeed, since $R(t)>0$ and $\rho_{k}(q, t)$ are non-negative functions, even with respect to $q$ variable, then by substitution $\xi=\sqrt{\Omega_{0}} R(t) q$, one has

$$
\int_{-\infty}^{\infty} \rho_{k}(q, t) d q=\lim _{M->\infty} \int_{-M}^{M} \rho_{k}(q, t) d q=\lim _{M->\infty} \frac{1}{2^{k} k ! \sqrt{\pi}} \int_{-M \sqrt{\Omega_{0}} R(t)}^{M \sqrt{\Omega_{0}} R(t)} \exp \left(-\xi^{2}\right) H_{k}^{2}(\xi) d \xi=1,
$$

for any $t$ and each $k$, as consequence of the well known integral $\int_{-\infty}^{\infty} \exp \left(-\xi^{2}\right) H_{k}^{2}(\xi) d \xi=2^{k} k ! \sqrt{\pi}$. Thus, for any $M>0$, one has $\int_{-M}^{M} \rho_{k}(q, t) d q \leq \int_{-\infty}^{\infty} \rho_{k}(q, t) d q=1$, which shows that the integral $\int_{-M}^{M} \rho_{k}(q, t) d q$ is bounded by a constant independent on $M$ and $t$. On the other hand, for any $M>0$ and each $k=0,1,2, \ldots$,

$$
\lim _{t \rightarrow \infty} \int_{-M}^{M} \rho_{k}(q, t) d q=\lim _{t \rightarrow>\infty} \frac{1}{2^{k} k ! \sqrt{\pi}} \int_{-M \sqrt{\Omega_{0}} R(t)}^{M \sqrt{\Omega_{0}} R(t)} \exp \left(-\xi^{2}\right) H_{k}^{2}(\xi) d \xi=1,
$$

and since

$$
\int_{-\infty}^{\infty} \rho_{k}(q, t) d q=\int_{-\infty}^{-M} \rho_{k}(q, t) d q+\int_{-M}^{M} \rho_{k}(q, t) d q+\int_{M}^{\infty} \rho_{k}(q, t) d q=1
$$

one has

$$
\lim _{t \rightarrow \infty} \int_{-\infty}^{-M} \rho_{k}(q, t) d q=0 \quad \text { and } \quad \lim _{t \rightarrow \infty} \int_{M}^{\infty} \rho_{k}(q, t) d q=0 .
$$

Then, according to the definition of delta-convergent sequence, see Ref. 17, the result (67) follows. Another approach to see that $\rho_{k}(q, t)$ converges to Dirac delta is to show that,

$$
\lim _{t \rightarrow \infty} \int_{-\infty}^{\infty} \rho_{k}(q, t) f(q) d q=f(0),
$$

for test functions $f$ on $R$, (infinitely differentiable function vanishing outside of some bounded interval). In fact, using that (68) holds for every $t$, one can write

$$
\int_{-\infty}^{\infty} \rho_{k}(q, t) f(q) d q-f(0)=\int_{-\infty}^{\infty} \rho_{k}(q, t)[f(q)-f(0)] d q .
$$

Then, if $[-M, M]$ contains the support of $f$, that is $f(q)=0$ for $|q| \geq M$, one has

$$
\begin{aligned}
\left|\int_{-\infty}^{\infty} \rho_{k}(q, t) f(q) d q-f(0)\right| & \leq|f(0)| \int_{-\infty}^{-M} \rho_{k}(q, t) d q \\
& +\int_{-M}^{M} \rho_{k}(q, t)|f(q)-f(0)| d q+|f(0)| \int_{M}^{\infty} \rho_{k}(q, t) d q,
\end{aligned}
$$


where by (70),

$$
\lim _{t \rightarrow \infty}|f(0)| \int_{-\infty}^{-M} \rho_{k}(q, t) d q=0, \quad \lim _{t \rightarrow \infty}|f(0)| \int_{M}^{\infty} \rho_{k}(q, t) d q=0 .
$$

Therefore, we need only to show that

$$
\lim _{t \rightarrow>\infty} \int_{-M}^{M} \rho_{k}(q, t)|f(q)-f(0)| d q=0 .
$$

By the mean-value theorem, one has $|f(q)-f(0)| \leq \max \left|f^{\prime}(q)\right||q|$, and thus

$\int_{-M}^{M} \rho_{k}(q, t)|f(q)-f(0)| d q \leq \max \left|f^{\prime}(q)\right| \int_{-M}^{M}|q| \rho_{k}(q, t) d q=2 \max \left|f^{\prime}(q)\right| \int_{0}^{M} q \rho_{k}(q, t) d q$.

To evaluate the last integral one can write explicitly:

$$
\begin{aligned}
\int_{0}^{M} q \rho_{k}(q, t) d q=\frac{1}{2^{k} k ! \sqrt{\pi}} \int_{0}^{M} & \sqrt{\Omega_{0}} R(t) q \exp \left(-\left(\sqrt{\Omega_{0}} R(t) q\right)^{2}\right) H_{k}^{2}\left(\sqrt{\Omega_{0}} R(t) q\right) d q \\
= & \left(\frac{1}{2^{k} k ! \sqrt{\pi}}\right) \frac{1}{\sqrt{\Omega_{0}} R(t)} \int_{0}^{M \sqrt{\Omega_{0}} R(t)} \xi \exp \left(-\xi^{2}\right) H_{k}^{2}(\xi) d \xi .
\end{aligned}
$$

Thus, it is not difficult to see that $\lim _{t->\infty} \int_{0}^{M} q \rho_{k}(q, t) d q=0$, from which (73) follows. Finally, from (72) and (73) the proof of (71) is done.

\section{CONCLUSION}

In the present paper, we have described time variable Madelung fluid and its linearization in terms of time variable linear Schrödinger equation. Our model, as descriptive of dissipative quantum fluid, admits exact solution for specific time-dependent systems, like the harmonic oscillator with time-dependent frequency and mass. In this case, exact time evolution has been described in terms of solutions for the corresponding damped classical oscillator. In particular, for the damping simulated by an exponentially growing mass (the Caldirola-Kanai model), it was shown that the quantum damping squeezes the density function of the fluid and leads to Dirac-delta function. This can have some implications in quantum cosmology. In fact, if $\Psi(q, t)$ is a solution of the Caldirola-Kanai oscillator for a damped system

$$
i \hbar \frac{\partial \Psi}{\partial t}=-\frac{\hbar^{2}}{2} e^{-\gamma t} \frac{\partial^{2} \Psi}{\partial q^{2}}+\frac{1}{2} \omega_{0}^{2} e^{\gamma t} q^{2} \Psi
$$

then $\Phi(q, t)=\Psi^{*}(q,-t)$, where $(*)$ denotes complex conjugation, is a solution of the related dual amplified system:

$$
i \hbar \frac{\partial \Phi}{\partial t}=-\frac{\hbar^{2}}{2} e^{\gamma t} \frac{\partial^{2} \Phi}{\partial q^{2}}+\frac{1}{2} \omega_{0}^{2} e^{-\gamma t} q^{2} \Phi .
$$

Hence, knowing that the solution $\Psi(q, t)$ of the Caldirola-Kanai model has merging zeros and describes collapse of the wave function to Dirac-delta function at time infinity, leads to possible interpretation of the solution $\Psi^{*}(q,-t)$ for the dual system. Namely, we will have expanding wave function with creation of zeros as point particles from initial singularity at time zero. And this evolution simulates quantum mechanism similar to creation of expanding Universe from initial singularity in Big-Bang cosmology.

Finally, we note that by our results it is possible to find explicit exact solutions to a wide class of exactly solvable dissipative quantum fluids and complex Schrödinger-Burgers equations. For this we can use our recent work on exactly solvable dissipative systems, such as quantum Sturm-Liouville problems (Ref. 9). Then, it is possible also to describe the dynamics of the zeros and poles in the corresponding dissipative linear and nonlinear systems. These questions are under investigation now. 


\section{ACKNOWLEDGMENTS}

This work was partially supported by Izmir Institute of Technology, Turkey.

${ }^{1}$ V. E. Zakharov, "Dispersionless limit of integrable systems in $2+1$ dimensions," in Singular Limit of Dispersive Waves, edited by N. M. Ercolani, I. R. Gabitov, C. D. Levermore, and D. Serre (Plenum, New York, 1994), Vol. 320.

${ }^{2}$ E. Madelung, Z. Phys. 40, 322 (1926).

${ }^{3}$ P. R. Holland, The Quantum Theory of Motion (Cambridge University Press, Cambridge, England, 1993).

${ }^{4}$ R. Feynman, The Feynman Lectures on Physics, Quantum Mechanics (Addison-Wesley, Reading, MA, 1965), Vol. 3.

${ }^{5}$ R. Feynman, Statistical Mechanics, 2nd ed. (Westview Press, Boulder, Colorado, 1998).

${ }^{6}$ J. D. Cole, Q. Appl. Math. 9, 225 (1951).

${ }^{7}$ E. Hopf, Commun. Pure Appl. Math. 3, 201 (1950).

${ }^{8}$ F. Calogero, in Nonlinear Equations in Physics and Mathematics, Lett. Nuovo Cim. B 43 (1978) 177-241; in Nonlinear equations in physics and mathematics Vol. B 43, edited by Barut A. O

${ }^{9}$ Ş. A. Büyükaşık, O. K. Pashaev, and E. Ulaş-Tigrak, J. Math. Phys. 50, 072102 (2009).

${ }_{10}$ J. Wei and E. Norman, J. Math. Phys. 4, 575 (1963).

${ }^{11}$ A. Perelomov, Generalized Goherent States and Their Applications (Springer-Verlag, Berlin, 1986).

${ }^{12}$ G. Dattoli, S. Solimeno, and A. Torre, Phys. Rev. A 34, 2646 (1986).

${ }^{13}$ P. Caldirola, Nouovo Cimento 18, 393 (1941).

${ }^{14}$ E. Kanai, Prog. Theor. Physics 3, 440 (1948).

${ }^{15}$ G. Dattoli, P. L. Ottaviani, A. Torre, and L. Vazquez, Rivista Del Nuovo Cimento 20, N.2 (1997).

${ }^{16}$ E. A. Galapon, J. Phys. A 42, 175201 (2009).

${ }^{17}$ I. M. Gelfand and G. E. Shilov, Generalized Functions (Academic, New York, 1964), Vol. 1.

${ }^{18}$ R. R. Parwani and O. K. Pashaev, J. Phys. A 41, 235207 (2008).

${ }^{19}$ O. K. Pashaev and J.-H. Lee, Abelian Gauge Theory and Integrable Sigma Models (Institute of Mathematics, Academia Sinica, Taiwan, 2000).

${ }^{20}$ O. K. Pashaev, Teoret. Mat. Fiz. 160, 178 (2009). 